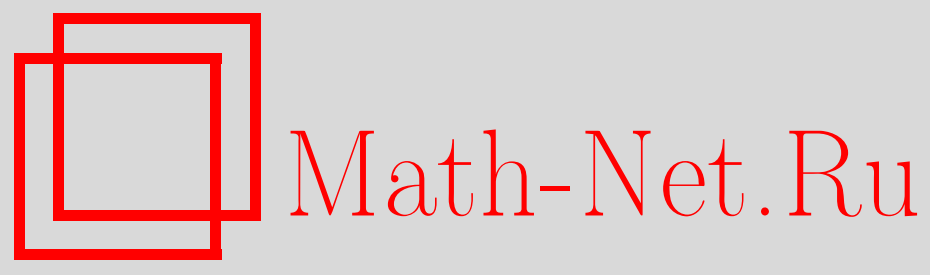

Л. Г. Бабат, А. А. Фридман, Параллельные вложения октаэдрических многогранников, Дискрет. матем., 2008, том 20, выпуск 2, 122-159

DOI: https://doi.org/10.4213/dm1007

Использование Общероссийского математического портала Math-Net.Ru подразумевает, что вы прочитали и согласны с пользовательским соглашением http://www.mathnet.ru/rus/agreement

Параметры загрузки:

IP: 54.84 .234 .179

26 апреля 2023 г., $17: 02: 38$ 


\title{
Параллельные вложения октаэдрических многогранников
}

\author{
(ㄷ 2008 г. Л. Г. Бабат, А. А. Фридман
}

Построено комбинаторное представление октаэдрического многогранника (кристалла), найдены его геометрические параметры, и показано, что параметры такого многогранника и его зеркального отображения совпадают и просто измеряются.

С использованием комбинаторного представления алмаза построен алгоритм, который находит круглый бриллиант максимального радиуса, вложимый в октаэдрический алмаз, и определяет местоположение такого бриллианта в алмазе. Все это привело к созданию технологического процесса переработки октаэдрических алмазов в круглые бриллианты максимальной стоимости.

\section{1. Введение}

В 1955-1956 гг. в СССР были найдены промышленные месторождения алмазов, и в короткий срок страна стала одним из крупнейших в мире производителей алмазов и бриллиантов - источников свободно конвертируемой валюты (до 2004 г. данные о добыче алмазов, производстве бриллиантов в нашей стране и торговле ими были засекречены, их публикация началась в 2005 г. после принятия соответствующего Указа Президента РФ). Алмаз является стратегическим сырьем высочайшей удельной ценности. Достаточно сказать, что алмазы являются индикаторами научно-технического прогресса, причем цена высококачественного алмаза весом в 1 карат (0,2 г) в 1000 раз выше цены 1 карата золота, а цена хорошего бриллианта весом в 1 карат превосходит ее уже в 5-8 тысяч раз. Естественно встала проблема эффективного использования алмазов. В первую очередь она возникла для производства круглых бриллиантов, доля которых в мировом производстве около 85\%, и для высококачественных алмазов. Круглый бриллиант представляет собой тело, похожее на юлу, причем параметры его формы регламентированы и однозначно определяются по его диаметру. Соответствующие исследования этой проблемы велись давно, но только недавно стала возможной открытая публикация полученных результатов.

Высококачественный алмаз представляет собой восьмигранник, который можно получить из правильного октаэдра (идеального кристалла алмаза), смещая какие-либо из его граней параллельно самим себе. В дальнейшем такие многогранники (и алмазы) будем называть октаэдрическими.

Важно отметить, что цена алмаза и бриллианта (при прочих равных условиях) растет с увеличением их веса, причем при переходе через границы фиксированных весовых интервалов, этот рост имеет скачкообразный характер. В силу этого особое значение имеет задача получения (производства) из кристалла алмаза круглого бриллианта максимального размера (радиуса). 
Если бриллиант можно получить из алмаза, то этот бриллиант вкладывается в алмаз. Возникает задача вложения. Представим себе, что бриллиант $B$ вложен в октаэдрический алмаз (многогранник) $A$. Подвинем каждую из 8 граней $A$ параллельно самой себе до касания с $B$. Получится меньший октаэдрический многогранник $A_{B}$, вложенный в $A$ так, что его грани параллельны соответствующим граням $A$. Такое вложение октаэдрических многогранников будем называть параллельным. Возникает задача о параллельном вложении (вложимости) октаэдрического многогранника в другой октаэдрический многогранник.

Круглый бриллиант - фигура зеркально симметричная. Поэтому с точки зрения получения из алмаза $A$ круглого бриллианта $B$ возможности параллельного вложения в $A$ многогранника $A_{B}$ и зеркального отражения многогранника $A_{B}$ эквивалентны друг другу. В связи с этим задача о параллельном вложении октаэдрического многогранника в другой октаэдрический многогранник естественным образом превращается в задачу о параллельном вложении октаэдрического многогранника или его зеркального отражения в другой октаэдрический многогранник (в задачу о параллельном вложении с точностью до зеркального отражения).

При этом важно найти не только критерий возможности такого вложения октаэдрических многогранников, но и сформулировать его в достаточно простых терминах, характеризующих многогранник, как трехмерное геометрическое тело. Более того, чтобы решение задачи имело прикладное значение при производстве бриллиантов, параметры, используемые в критерии, должны не только естественно характеризовать многогранник, но и просто по нему измеряться. При этом параметры алмаза должны измеряться с высокой точностью (до сотых долей миллиметра). Ошибки в измерениях могут вызвать резкое расхождение оценочной и реальной стоимости получаемой из алмаза продукции. В противном случае результат останется чисто теоретическим, поскольку кристаллы алмаза обрабатываются индивидуально, а число ежедневно обрабатываемых кристаллов исчисляется многими тысячами.

В процессе анализа и решения этой задачи удалось построить так называемое комбинаторное представление октаэдрического многогранника (кристалла), найти его геометрические параметры, показать, что параметры такого многогранника и его зеркального отображения совпадают и просто измеряются, уточнить формулировку задачи, найти критерий параллельной вложимости (с точностью до зеркального отражения), доказав теоремы 1 и 2.

Опираясь на теорему 2 и используя комбинаторное представление алмаза, удалось построить алгоритм, который находит круглый бриллиант максимального радиуса, вложимый в октаэдрический алмаз, и определяет местоположение такого бриллианта в алмазе. Все это привело к созданию технологического процесса переработки октаэдрических алмазов в круглые бриллианты максимальной стоимости.

Естественно возник вопрос об использовании полученных результатов для оценивания алмазов с точки зрения стоимости получаемых из них бриллиантов. Выяснилось, что геометрия октаэдрического алмаза (соотношение его линейных параметров) влияет на стоимость получаемых из него круглых бриллиантов, не меньше чем вес алмаза, то есть фактор, на котором основывались вековые стереотипы оценки. Эти стереотипы оказались неверны. Полученные результаты позволили построить примеры, когда

- из октаэдрического алмаза меньшего веса получается круглый бриллиант большего диаметра, чем из октаэдрического алмаза большего веса;

- пары октаэдрических алмазов, неразличимые с точки зрения первоклассных оценщиков (то есть практически одинаковые), отличаются по стоимости получаемых из них круглых бриллиантов в 1,5 раза, что при громадной стоимости алмазов 
и бриллиантов весьма существенно (различие линейных размеров таких алмазов составляет не более чем $0,5 \%$ по любому из параметров, лежащих в диапазоне 4-12 мм).

Примеры поразили специалистов. Одна из причин этого - сложившееся у них представление, что обработка и оценка алмазов основывается исключительно на человеческой интуиции и передаваемом из поколения в поколение индивидуальном опыте.

Настоящая статья посвящена комбинаторному описанию и исследованию октаэдрических многогранников и доказательству теоремы о критерии параллельного вложения с точностью до зеркального отражения таких многогранников друг в друга.

\section{2. Октаэдрические наборы и звезды}

Здесь вводятся комбинаторные объекты, в терминах которых характеризуются октаэдрические многогранники, и формулируется теорема о параллельном вложении.

Определение 1. Назовем н-гранью (нормаль-гранью) пару $\{\alpha ; \vec{A}\}$, где $\alpha-$ плоскость, а $\vec{A}-$ выходящая из точки этой плоскости ее нормаль. Плоскость $\alpha$ делит пространство на два открытых полупространства, $H_{+}$, куда направлен вектор $\vec{A}$ и $H_{-A}$, куда направлен вектор $-\vec{A}$. Объединение $\alpha \cup H_{-A}$ (то есть замыкание полупространства $H_{-A}$ ) назовем собственным полупространством н-грани $\{\alpha ; \vec{A}\}$.

Определение 2. Пусть $M$ - выпуклый многогранник, $\vec{N}_{1}, \vec{N}_{2}, \ldots, \vec{N}_{k}$ - внешние нормали его граней, а $\gamma_{1}, \gamma_{2}, \ldots, \gamma_{k}-$ плоскости, на которых лежат эти грани. Совокупность н-граней $\left\{\gamma_{1} ; \vec{F}_{1}\right\},\left\{\gamma_{2} ; \vec{F}_{2}\right\}, \ldots,\left\{\gamma_{k} ; \vec{F}_{k}\right\}$, где $\vec{F}_{j}=\vec{N}_{j}, j=1,2, \ldots, k$, назовем порождающим набором многогранника $M$.

Замечание 1. Вектор $\vec{F}_{j}$ может выходить из точки плоскости $\gamma_{j}$, не принадлежащей грани многогранника $M, j=1,2, \ldots, k$.

Используя введенные термины, придадим следующий вид трем известным фактам.

Предложение 1. Пусть $\left\{\gamma_{1} ; \vec{F}_{1}\right\},\left\{\gamma_{2} ; \vec{F}_{2}\right\}, \ldots,\left\{\gamma_{k} ; \vec{F}_{k}\right\}$ - порождающий набор выпуклого многогранника $M$, а $P_{j}-$ собственное полупространство н-грани $\left\{\gamma_{j} ; \vec{F}_{j}\right\}$, $j=1,2, \ldots, k$. Тогда $M$ совпадает с пересечением $P_{1} \cap P_{2} \cap \ldots \cap P_{k}$, причем внутренностью $М$ является пересечение $\left(P_{1} \backslash \gamma_{1}\right) \cap\left(P_{2} \backslash \gamma_{2}\right) \cap \ldots \cap\left(P_{k} \backslash \gamma_{k}\right)$.

Предложение 2. Если $A$-набор из $k$ н-граней, то

(а) пересечение P их собственных полупространств есть выпуклое многогранное множество, число граней которого не превосходит $k$;

(б) для каждой грани $G$ множества $P$ найдется такая н-грань $\{\alpha, \vec{V}\} \in A$, что $G \subset \alpha$, а внешняя нормаль $\vec{N}$ грани $G$ удовлетворяет условию $\vec{N}=c \vec{V}$, где $c>0$.

Замечание 2. Пересечение $P$ не обязательно является многогранником. $P$ может быть неограниченным или вырожденным (двумерным, одномерным, точкой, пустым). Утверждается только выпуклость $P$ и дается оценка числа граней $P$ для случая, когда оно трехмерно. Для вырожденных ситуаций говорить о числе граней $P$ в трехмерном пространстве бессмысленно и потому считается, что в этих ситуациях у $P$ имеется 0 граней. 


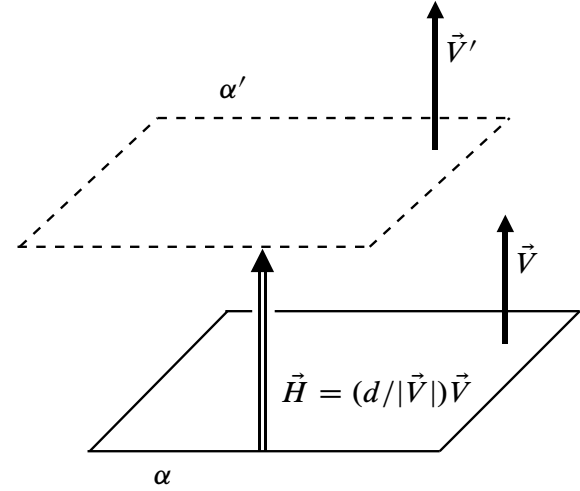

Фиг. 1

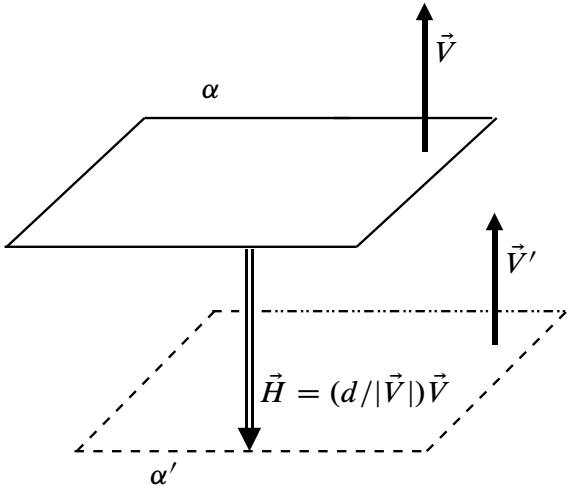

Фиг. 2

Pис. 1.

Напомним, что евклидовым называется линейное аффинное преобразование, сохраняющее модуль скалярного произведения (расстояние). Под гомотетией всегда будем подразумевать гомотетию с ненулевым коэффициентом.

Предложение 3. Пусть $\left\{\alpha_{1} ; \vec{A}_{1}\right\},\left\{\alpha_{2} ; \vec{A}_{2}\right\}, \ldots,\left\{\alpha_{n} ; \vec{A}_{n}\right\}-$-грани, a $P_{1}, \ldots, P_{n}-u x$ собственные полупространства. Если $E$ - евклидово преобразование или гомотетия, то nары $\left\{E\left(\alpha_{1}\right) ; E\left(\vec{A}_{1}\right)\right\},\left\{E\left(\alpha_{2}\right) ; E\left(\vec{A}_{2}\right)\right\}, \ldots,\left\{E\left(\alpha_{n}\right) ; E\left(\vec{A}_{n}\right)\right\}$ также являются н-гранями, причем

(а) собственные полупространства н-граней $\left\{E\left(\alpha_{1}\right) ; E\left(\vec{A}_{1}\right)\right\},\left\{E\left(\alpha_{2}\right) ; E\left(\vec{A}_{2}\right)\right\}, \ldots$, $\left\{E\left(\alpha_{n}\right) ; E\left(\vec{A}_{n}\right)\right\}-$ это $E\left(P_{1}\right), \ldots, E\left(P_{n}\right)$;

(б) $E\left(P_{1} \cap P_{2} \cap \ldots \cap P_{n}\right)=E\left(P_{1}\right) \cap \ldots \cap E\left(P_{n}\right)$;

(в) число граней и размерности множеств $P_{n} \cap \ldots \cap P_{n} u E\left(P_{1} \cap \ldots \cap P_{n}\right)$ совпадают.

Нужные нам свойства октаэдрических многогранников удобно формулировать и анализировать в терминах н-граней и порождающих наборов. Поэтому сформулируем в этих терминах определение октаэдрических многогранников. Для этого введем следующую операцию над н-гранями.

Определение 3. Рассмотрим н-грань $\{\alpha ; \vec{V}\}$, число $d$ и вектор $\vec{H}=d \vec{V} /|\vec{V}|$, вектор $\vec{H}$ удовлетворяет трем условиям:

(a) его длина $|\vec{H}|$ равна $d$;

(б) при $d>0$ вектор $\vec{H}$ направлен в ту же сторону, что и вектор $\vec{V}$;

(в) при $d<0$ вектор $\vec{H}$ направлен в сторону, противоположную $\vec{V}$.

Назовем $d$-смещением н-грани $\{\alpha ; \vec{V}\}$ н-грань $\left\{\alpha^{\prime} ; \vec{V}^{\prime}\right\}$, где $\alpha^{\prime}-$ плоскость, получающаяся из $\alpha$, параллельным сдвигом на $\vec{H}$, а $\vec{V}^{\prime}-$ нормаль к $\alpha^{\prime}$, равная $\vec{V}$ (см. рис. 1 , где на фиг. 1 изображена ситуация $d>0$, а на фиг. $2-$ ситуация $d<0$ ). 
Замечание 3. Рассматривая $d$-смещение н-грани, будем использовать упрощенную терминологию: вместо слов, что существует $d \leqslant q$ такое, что н-грань $\left\{\alpha^{\prime} ; \vec{V}^{\prime}\right\}$ есть $d$-смещение н-грани $\{\alpha ; \vec{V}\}$, будем говорить, что н-грань $\left\{\alpha^{\prime} ; \vec{V}^{\prime}\right\}$ есть смещение н-грани $\{\alpha ; \vec{V}\}$ на величину, не превосходящую $q$. Если же ограничение $d \leqslant q$ несущественно, будем просто говорить, что н-грань $\left\{\alpha^{\prime} ; \vec{V}^{\prime}\right\}$ есть смещение н-грани $\{\alpha ; \vec{V}\}$.

Нам потребуется также ряд понятий, относящихся к наборам н-граней.

Определение 4. Набор $A$ из $k$ н-граней назовем невырожденным, если пересечение собственных полупространств его н-граней есть трехмерное многогранное множество, имеющее ровно $k$ граней (ср. предложение 2).

Определение 5. Пусть $A$ - невырожденный набор н-граней. Скажем, что набор н-граней $B$ похож на набор $A$, если:

(a) $B$ получается из $A$ заменой ровно одной его н-грани;

(б) заменяющая грань $\{\beta ; \vec{N}\}$ есть смещение заменяемой грани $\{\alpha ; \vec{F}\}$;

(в) пересечение $P_{A}$ собственных полупространств граней из набора $A$ имеет общую точку с пересечением $P_{B}$ собственных полупространств н-граней из набора $B$.

Замечание 4. Подчеркнем, что набор $A$, на который похож набор $B$, обязательно невырожденный. На $B$ такое требование не налагается. Поэтому отношение похожести, вообще говоря, не является симметричным. Кроме того из п. (а) следует, что $\alpha \| \beta$. Соотношение $P_{A} \cap P_{B} \neq \varnothing$ означает, что если плоскость $\alpha$ сдвигать параллельно самой себе, пока она не перейдет в плоскость $\beta$, то в $P_{A}$ найдется точка $T$, которая при таком движении плоскости $\alpha$ не пройдет сквозь нее (то есть $\alpha$ либо не достигнет данной точки, либо остановится, когда $T$ попадет на нее).

Определение 6. Набор $A$ н-граней назовем регулярным, если можно указать натуральное $n$ и цепочку из $n$ наборов н-граней

$$
T_{1} \rightarrow T_{2} \rightarrow \ldots \rightarrow T_{n}=A
$$

удовлетворяющую условиям:

(a) набор $T_{1}$ есть порождающий набор правильного многогранника;

(б) набор $T_{i+1}$ похож на набор $T_{i}, i=1, \ldots, n-1$.

Замечание 5. Так как набор н-граней может быть похож только на невырожденный набор н-граней, то наборы $T_{1}, \ldots, T_{n-1}$ из цепочки (1) невырожденные. Набор $T_{n}$ может быть вырожденным.

Среди регулярных наборов н-граней выделим наборы двух видов - октаэдрические и тетраэдрические.

Определение 7. Набор н-граней назовем октаэдрическим (соответственно тетраэдрическим) если он регулярный и состоит из 8 (соответственно из 4) граней.

Замечание 6. Это определение означает, что цепочка вида (1), приводящая к октаэдрическому набору, начинается с порождающего набора правильного октаэдра, а приводящая к тетраэдрическому набору - с порождающего набора правильного тетраэдра. 

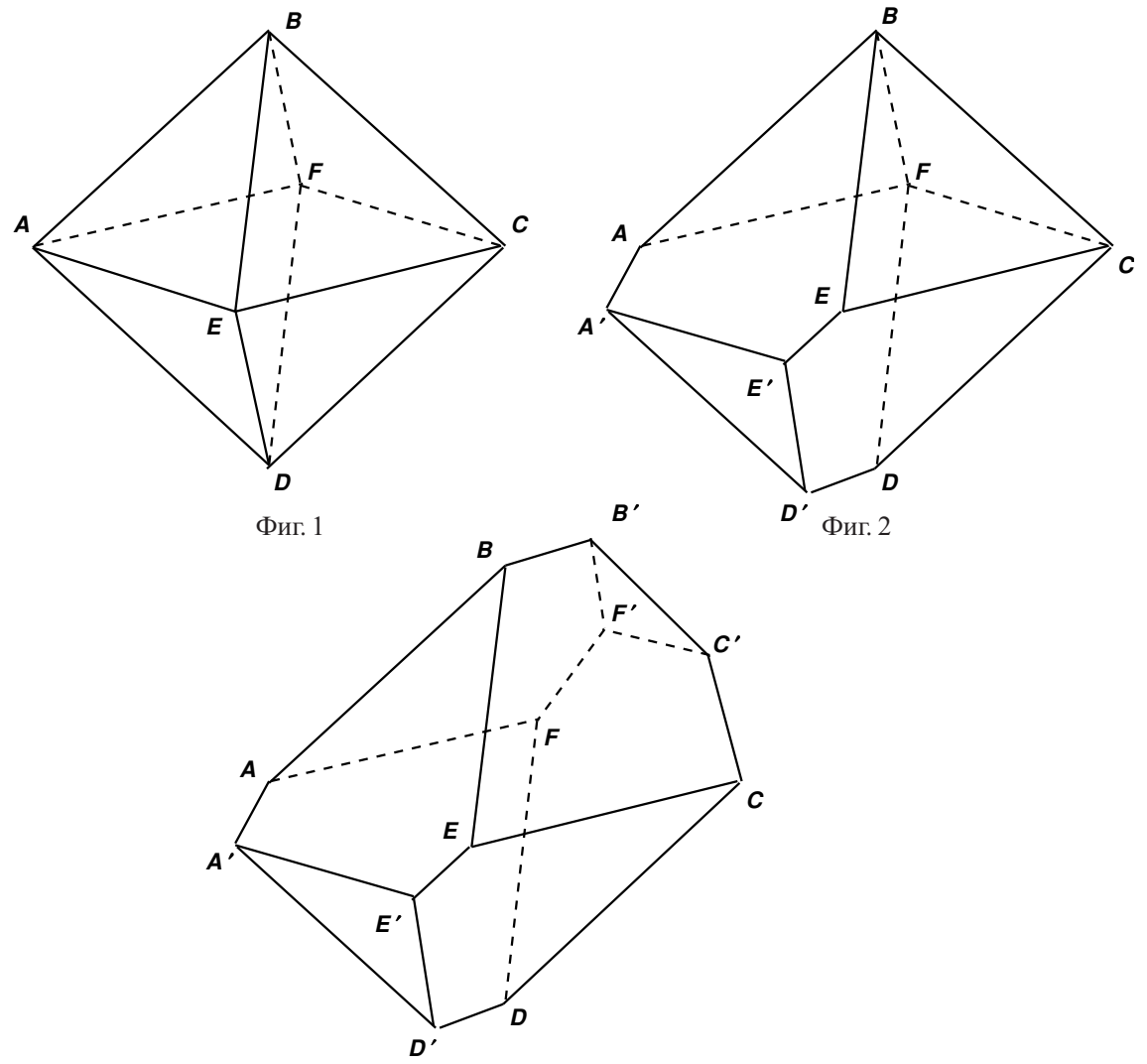

Фиг. 3

Pис. 2.

Используя понятие октаэдрического набора, сформулируем описание октаэдрических многогранников в терминах н-граней и порождающих наборов.

Определение 8. Многогранник $M$ - октаэдрический, если он есть пересечение собственных полупространств н-граней октаэдрического набора.

Для наглядности приведем на рис. 2 примеры октаэдрических многогранников, получающихся друг из друга сдвигом вовне сначала плоскости грани $A E D$, а потом плоскости грани $B F C$. Многогранник, изображенный на фиг. 3, является типичным примером октаэдрического многогранника.

Возникает два вопроса. Первый: зачем введено общее понятие регулярного набора н-граней, когда с интересующими нас алмазами связаны только октаэдрические наборы. Второй: почему, выделяя среди регулярных наборов октаэдрические, мы не ограничились невырожденными наборами, связанными с интересующими нас алмазами. Ответ на оба вопроса один. Многие построения и доказательства получаются нагляднее и короче, если их проводить, выходя за класс невырожденных октаэдрических наборов.

Напомним, что основная цель работы - нахождение критериев возможности параллельного вложения октаэдрических многогранников. Задав такие многогранники в терминах регулярных наборов н-граней, надо задать в этих же терминах понятие параллельного вложения. Для этого сначала определим действие евклидовых преобразований на регулярные наборы н-граней. 
Определение 9. Пусть $E$ - евклидово преобразование или гомотетия. Образом н-грани $\{\gamma ; \vec{N}\}$ при преобразовании $E$ назовем н-грань $\{E(\gamma) ; E(\vec{N})\}$, а образом набора н-граней $A-$ набор $E(A)$ образов н-граней набора $A$.

Замечание 7. Корректность этого определения вытекает из предложения 3.

Относительно несложно показать, что имеют место следующие две леммы, которые устанавливают замкнутость относительно евклидовых преобразований и гомотетий класса регулярных наборов н-граней, а также его подклассов, состоящих из октаэдрических и тетраэдрических наборов.

Лемма 1. Пусть $E-$ евклидово преобразование или гомотетия. Если набор $B$ н-граней похож на набор А н-граней, то набор граней $E(B)$ похож на набор граней $E(A)$.

Лемма 2. Если $A$ - регулярный набор н-граней, а $E$ - евклидово преобразование или гомотетия, то набор н-граней $E(A)$ - регулярный, и число его н-граней равно числу н-граней набора $A$.

Теперь можно определить, когда один набор н-граней вложен в другой и когда один набор н-граней вкладывается (вложим) в другой.

Определение 10. Пусть $A$ и $B$ - наборы н-граней. Скажем, что набор $A$ параллельно вложен в набор $B$, если между $A$ и $B$ можно установить взаимно однозначное соответствие, при котором любые сопоставленные друг другу грани $\{\mu ; \vec{F}\} \in A$ и $\{\gamma ; \vec{N}\} \in B$ удовлетворяют условиям:

(a) $\vec{F}=c \vec{N}$, где $c>0$ (плоскости $\mu$ и $\gamma$ параллельны, а векторы $\vec{F}$ и $\vec{N}$ направлены в одну сторону);

(б) собственное полупространство н-грани $\{\mu ; \vec{F}\} \in A$ лежит в собственном полупространстве н-грани $\{\gamma ; \vec{N}\} \in B$.

Определение 11. Набор н-граней $A$ параллельно вкладывается в набор н-граней $B$, если существует такая комбинация $E$ поворота и параллельного переноса, что набор н-граней $E(A)$ параллельно вложен в $B$.

Введенные понятия позволяют описать возможность параллельного вложения одного выпуклого многогранника в другой в терминах н-граней.

Определение 12. Скажем, что выпуклый многогранник $A$ параллельно вкладывается в выпуклый многогранник $B$, если порождающий набор многогранника $A$ параллельно вкладывается в порождающий набор многогранника $B$.

Таким образом, вопрос о параллельном вложении октаэдрических многогранников сводится к вопросу о параллельном вложении октаэдрических наборов.

Для дальнейшего исследования сформулируем в терминах н-граней два известных свойства параллельных плоскостей, связанных с делением плоскостью пространства на два полупространства.

Предложение 4. Пусть $\{\alpha ; \vec{A}\} u\{\beta ; \vec{B}\}-$-грани, причем $\vec{A}=c \vec{B}$, где $c<0$. Если $M_{A} u$ $M_{B}$ - собственные полупространства этих н-граней, а $E$ - замкнутьий слой полупространства, ограниченный плоскостями $\alpha$ и $\beta$, то есть только три взаимоисключающие ситуации (см. рис. 3): 

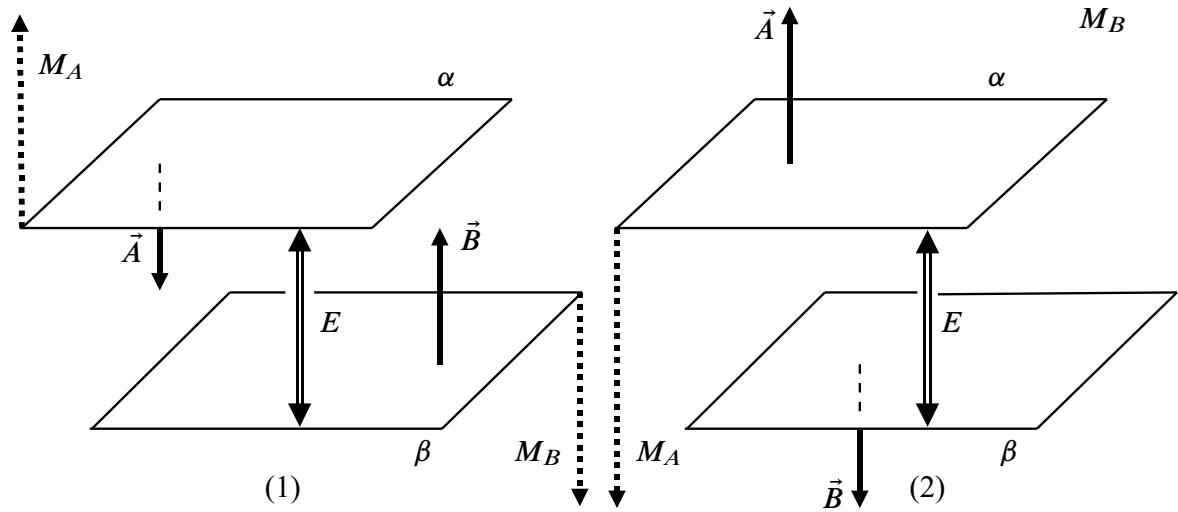

(1)

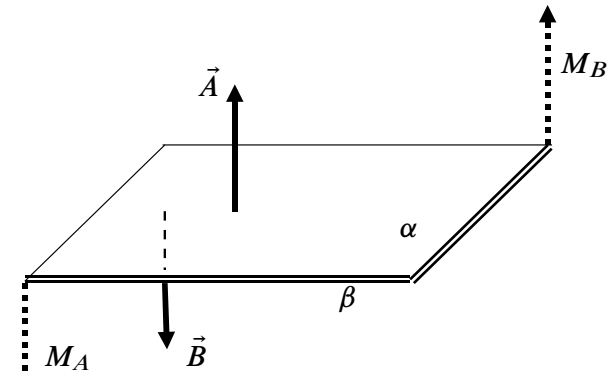

(3)

Рис. 3.

(1) $\alpha \neq \beta, \alpha \nsubseteq M_{B}, \beta \nsubseteq M_{A}, M_{A} \cap M_{B}=\varnothing$;

(2) $\alpha \neq \beta, \alpha \subset M_{B}, \beta \subset M_{A}, M_{A} \cap M_{B}=E$;

(3) $\alpha=\beta, M_{A} \cap M_{B}=E=\alpha=\beta$.

Предложение 5. Пусть $\{\alpha ; \vec{A}\} u\{\beta ; \vec{B}\}-$-грани, причем $\vec{A}=c \vec{B}$, где $c>0$. Если $M_{A} u$ $M_{B}$ - собственные полупространства этих н-граней, а $E$ - замкнутый слой полупространства, ограниченный плоскостями $\alpha$ и $\beta$, то есть только три взаимоисключаюшие ситуации (см. рис. 4):

(1) $\alpha \neq \beta, \alpha \subset M_{B}, M_{A} \subset M_{B}, E \cap\left(M_{A} \cap M_{B}\right)=\alpha$;

(2) $\alpha \neq \beta, \beta \subset M_{A}, E \cap\left(M_{A} \cap M_{B}\right)=\beta$;

(3) $\alpha=\beta, M_{A}=M_{B}, E=\alpha=\beta$.

Каждый октаэдрический набор удобно представлять, как пару особым образом связанных друг с другом тетраэдрических наборов. Далее такие пары будем называть звездами, для определения которых требуются промежуточные понятия.

Определение 13. Будем говорить, что тетраэдр $T$ индуцирован кубом $Q$, если на каждой грани куба $Q$ лежит одно ребро тетраэдра $T$, являющееся диагональю этой грани (см. рис. 5, где изображены два тетраэдра, индуцированные кубом $\left.A B^{*} B A^{*} D C^{*} C D^{*}\right)$. 

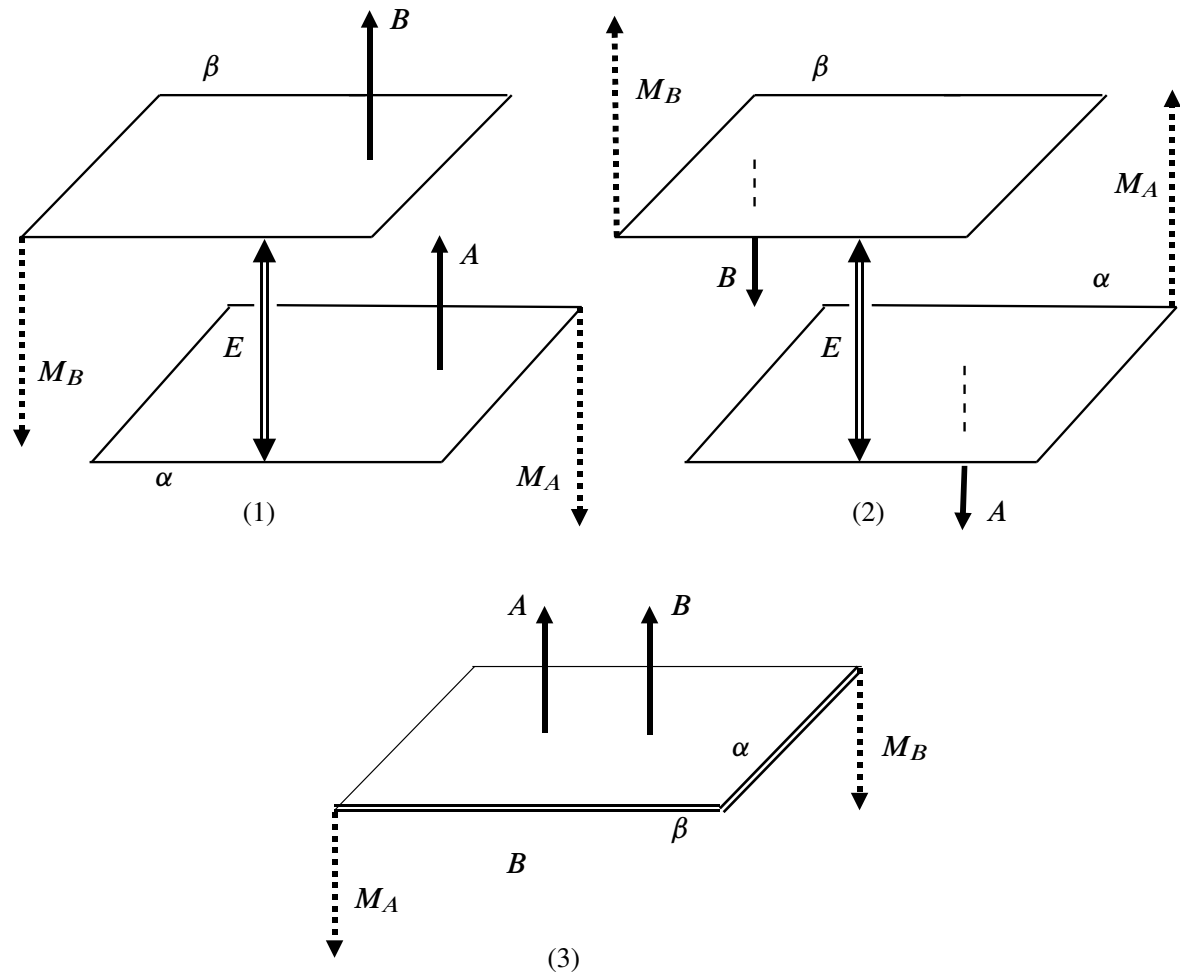

Рис. 4.
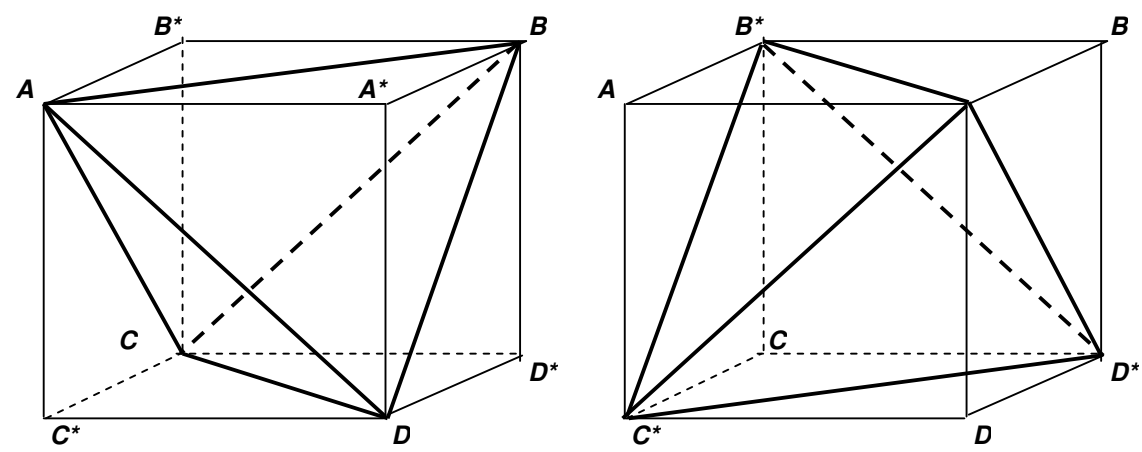

Рис. 5. 

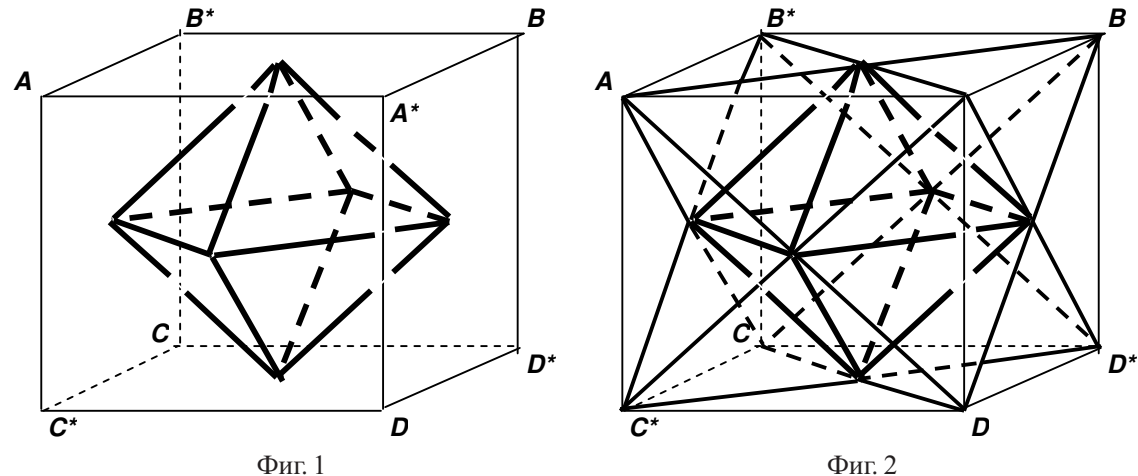

Фиг. 2

Рис. 6.

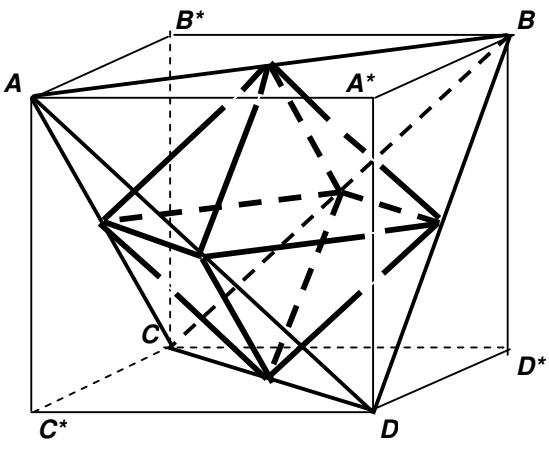

Фиг. 1

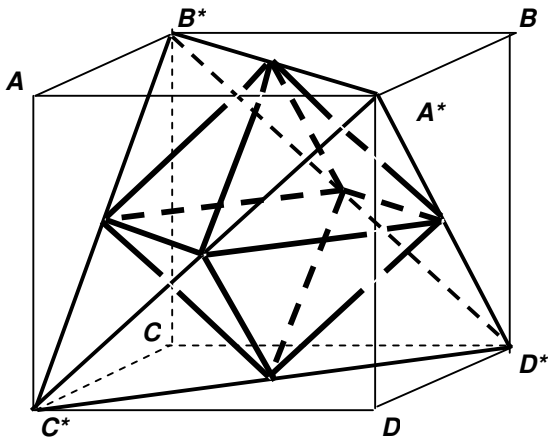

Фиг. 2

Рис. 7.

Пусть $G$ - грань куба $Q$. На $G$ есть ровно две диагонали. Значит существует ровно два способа расположения на грани $G$ ребра тетраэдра $T$, индуцированного кубом $Q$. Выбор положения ребра тетраэдра $T$ на грани $G$ однозначно определяет положение ребер данного тетраэдра на всех гранях куба $Q$. Поскольку диагонали граней куба равны между собой, каждый куб индуцирует ровно два тетраэдра, и оба они правильные (рис. 5). В связи с этим корректно следующее определение.

Определение 14. Идеальной звездой назовем пару $A, B$ тетраэдрических наборов н-граней, удовлетворяющих условию: имеется такой куб $Q$, что $A$ и $B$ есть порождающие наборы двух различных правильных тетраэдров, индуцированных кубом $Q$.

Ребра правильных тетраэдров, индуцированных кубом, пересекаются в центрах его граней. Поэтому пересечение тетраэдров, индуцированных кубом, есть многогранник с вершинами в центрах граней данного куба, то есть правильный октаэдр, расположенный в кубе так, как указано на фиг. 1 рис. 6. Положение октаэдра относительно обоих тетраэдров указано на фиг. 2 этого рисунка. Чтобы сделать эту фигуру более наглядной, на рис. 7 указано положение октаэдра относительно каждого из тетраэдров.

Пусть $\{A, B\}$ - идеальная звезда. Рассмотрим тетраэдры $T_{A}$ и $T_{B}$ с порождающими наборами $A$ и $B$. Так как противоположные грани правильного октаэдра $H=T_{A} \cap T_{B}$ 
параллельны, для каждой н-грани $\left\{\alpha ; \vec{V}_{A}\right\} \in A$ найдется такая н-грань $\left\{\beta ; \vec{V}_{B}\right\} \in B$, что $\alpha \| \beta$.При этом октаэдр $H$ лежит в пересечении собственных полупространств н-граней $\left\{\alpha ; \vec{V}_{A}\right\}$ и $\left\{\beta ; \vec{V}_{B}\right\}$. Поскольку правильный октаэдр имеет внутренние точки, очевидно,что для н-граней $\left\{\alpha ; \vec{V}_{A}\right\}$ и $\left\{\beta ; \vec{V}_{B}\right\}$ невозможна ни одна из ситуаций, перечисленных в предложении 5. Что касается ситуаций, перечисленных в предложении 4, то для этих граней возможна только ситуация 2. Поскольку ситуации, перечисленные в предложениях 4 и 5 , охватывают все возможные ситуации для н-граней $\left\{\alpha ; \vec{V}_{A}\right\}$ и $\left\{\beta ; \vec{V}_{B}\right\}$ при условии $\alpha \| \beta$, для этих н-граней выполняется ситуация 2 из предложения 4. Это означает, что векторы $\vec{V}_{A}$ и $\vec{V}_{B}$ коллинеарны и направлены в противоположные стороны. Поскольку любая грань правильного октаэдра параллельна только противоположной ей грани этого октаэдра, получаем такой факт.

Лемма 3. Если $\{A ; B\}-$-иеальная звезда, то для любой н-грани $\left\{\alpha ; \vec{V}_{A}\right\} \in A$ в В найдется ровно одна н-грань $\left\{\beta ; \vec{V}_{B}\right\} \in B$ такая, что $\alpha \| \beta$, при этом векторы $\vec{V}_{A}$ и $\vec{V}_{B}$ направлены в противоположные стороны (то есть, $\vec{V}_{A}=c \vec{V}_{B}$, где $\left.c<0\right)$.

Обратим внимание еще на одно не слишком сложно устанавливаемое свойство идеальных звезд.

Лемма 4. Пусть $\{A ; B\}-$ идеальная звезда, а $\left\{\alpha ; \vec{V}_{A}\right\} \in A u\left\{\beta ; \vec{V}_{B}\right\} \in B-$ ее н-грани. Если $\alpha \nmid \beta$, то угол между векторами $\vec{V}_{A} u \vec{V}_{B}$ равен $\arccos (1 / 3)$.

Напомним, что углом между двумя векторами принято считать тот из образуемых ими углов, который не превосходит $180^{\circ}$.

Для введения общего понятия звезды надо распространить отношение похожести, определенное для наборов н-граней (определение 5), на пары наборов н-граней.

Определение 15. Скажем, что пара $P$ из двух наборов н-граней похожа на пару $H$ из двух наборов н-граней, если между наборами из $H$ и наборами из $P$ можно установить такое взаимно однозначное соответствие, что

(a) одному набору из $H$ будет сопоставлен совпадающий с ним набор из $P$, а другому набору из $H-$ похожий на него набор из $P$;

(б) пересечение собственных полупространств н-граней из пары $H$ имеет общую точку с пересечением собственных полупространств н-граней из пары $P$.

Можно показать, что имеет место такой факт.

Лемма 5. Пусть $E$ - евклидово преобразование или гомотетия. Если пара наборов $\{A ; B\}$ н-граней похожа на пару наборов $\{C ; H\}$ н-граней, то пара наборов $\{E(A) ; E(B)\}$ н-граней похожа на пару наборов $\{E(C) ; E(H)\}$ н-граней.

Теперь можно дать определение понятия звезды.

Определение 16. Звездой назовем пару $\{A ; B\}$ наборов н-граней, для которой найдется натуральное $n$ и цепочка из $n$ пар наборов н-граней

$$
\left\{C_{1} ; D_{1}\right\} \rightarrow\left\{C_{2} ; D_{2}\right\} \rightarrow \ldots \rightarrow\left\{C_{n} ; D_{n}\right\}=\{A ; B\},
$$

удовлетворяющие условиям:

(a) $\left\{C_{1} ; D_{1}\right\}$ - идеальная звезда; 
(б) пара наборов $\left\{C_{i+1} ; D_{i+1}\right\}$ похожа на пару наборов $\left\{C_{i} ; D_{i}\right\}, i=1,2, \ldots, n-1$.

Для рассмотрения свойств звезд нам потребуется сформулированное в терминах н-граней и их смещений следующее известное свойство тетраэдров.

Предложение 6. Пусть $A B C D-$ тетраэдр с порождающчим набором $\left\{\alpha ; \vec{V}_{A}\right\},\left\{\beta ; \vec{V}_{B}\right\}$, $\left\{\sigma ; \vec{V}_{C}\right\},\left\{\delta ; \vec{V}_{C}\right\}$, где $\alpha, \beta, \sigma$ и $\delta-$ плоскости, содержашче грани тетраэдра, противолежашие соответственно вершинам $A, B, C$ и $D$. Обозначим $M_{A}, M_{B}, M_{C} u M_{D}$ собственные полупространства н-граней $\left\{\alpha ; \vec{V}_{A}\right\},\left\{\beta ; \vec{V}_{B}\right\},\left\{\sigma ; \vec{V}_{C}\right\} u\left\{\delta ; \vec{V}_{C}\right\}$ соответственно. Пусть расстояние от вериины А до плоскости $\alpha$ равно $h$. Рассмотрим число $d u$ $d$-смещение $\left\{\alpha^{\prime} ; \vec{V}_{A}^{\prime}\right\}$ н-грани $\left\{\alpha ; \vec{V}_{A}\right\}$. Если $M_{A}^{\prime}$ - собственное полупространство н-грани $\left\{\alpha^{\prime} ; \vec{V}_{A}^{\prime}\right\}$, a $P=M_{A}^{\prime} \cap M_{B} \cap M_{C} \cap M_{D}$, mo

(1) в случае $d>-h$ пересечение $P$ содержит хотя бы одну внутреннюю точку пересечения $P=M_{A} \cap M_{B} \cap M_{C} \cap M_{D}$ (внутреннюю точку тетраэдра $\left.A B C D\right)$, при этом $P$ будет тетраэдром, гомотетичным тетраэдру $A B C D$ с иентром гомотетии 6 его вериине $A$ и с коэффициентом гомотетии $(h+d) / h$;

(2) в случае $d=-h$ пересечение $P$ состоит из единственной точки - вериины $A$ исходного тетраэдра, через которую пройдет плоскость $\alpha^{\prime}$;

(3) в случае $d<-$ пересечение $P$ будет пустылм.

Для наглядности проиллюстрируем это свойство на рис. 8, где изображены ситуации, соответствующие четырем соотношениям величин $d$ и $h$ (случай 1 разделен на две ситуации $d \geqslant 0$ и $0>d>-h$ ). На каждой фигуре этих рисунков жирными линиями изображен исходный тетраэдр $A B C D$. Точечными стрелками указано, по какую сторону от плоскостей $\alpha$ и $\alpha^{\prime}$ расположены собственные полупространства $M_{A}$ и $M_{A}^{\prime}$ н-граней $\left\{\alpha ; \vec{V}_{A}\right\}$ и $\left\{\alpha^{\prime} ; \vec{V}_{A}^{\prime}\right\}$. Двойной стрелкой указан вектор, на который смещается параллельно самой себе плоскость $\alpha$. При этом

- на фиг. 1 изображена ситуация, когда $d \geqslant 0$, то есть когда пересечение $P$ есть тетраэдр $A^{\prime} B^{\prime} C^{\prime} D^{\prime}$, частью которого является исходный тетраэдр $A B C D$;

- на фиг. 2 изображена ситуация, когда $0>d>-h$, то есть когда пересечение $P$ есть тетраэдр $A^{\prime} B^{\prime} C^{\prime} D^{\prime}$, являющийся частью исходного тетраэдра $A B C D$;

- на фиг. 3 изображена ситуация, когда $d=-h$, то есть когда пересечение $P$ состоит из единственной точки - вершины $A$ исходного тетраэдра $A B C D$;

- на фиг. 4 изображена ситуация, когда $d<-h$, то есть пересечение $P$ пусто.

Из этого свойства тетраэдров вытекает важный факт о тетраэдрических наборах н-граней.

Лемма 6. Любой тетраэдрический набор н-граней удовлетворяет следующему условию: либо он является порождающим набором правильного тетраэдра, либо пересечение собственных полупространств его н-граней состочт ровно из одной точки, являющейся пересечением плоскостей, входящих в эти н-грани.

Доказательство. Если длина цепочки (1) равна 1, то доказываемое утверждение следует из первого пункта определения 6 и замечания 6. Пусть оно верно, когда длина цепочки (1) равна $k$. Покажем что тогда оно верно и для цепочки (1) длины $k+1$. Набор $T_{k}$ 

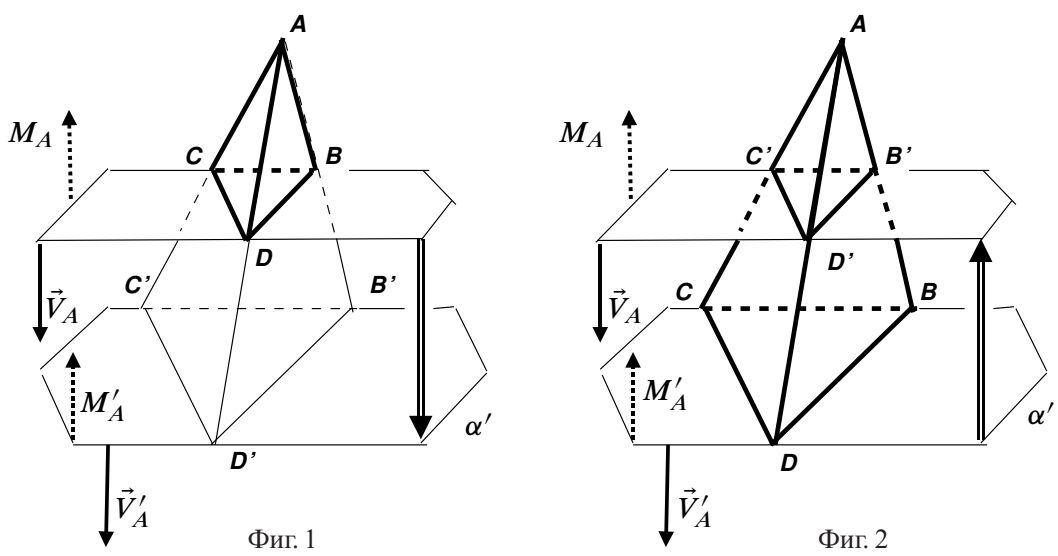

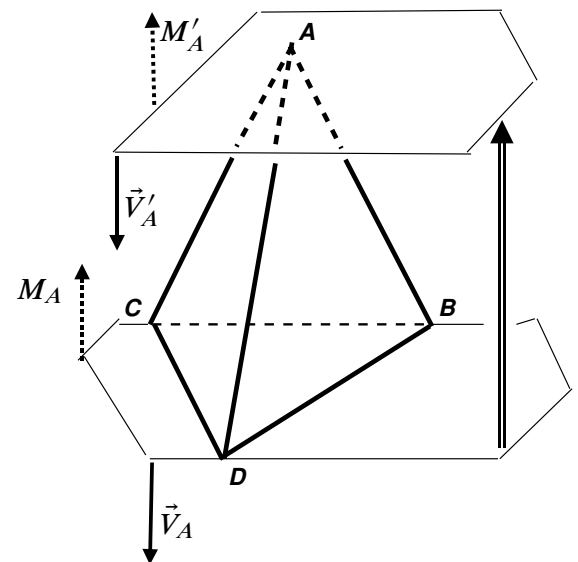

Фиг. 3

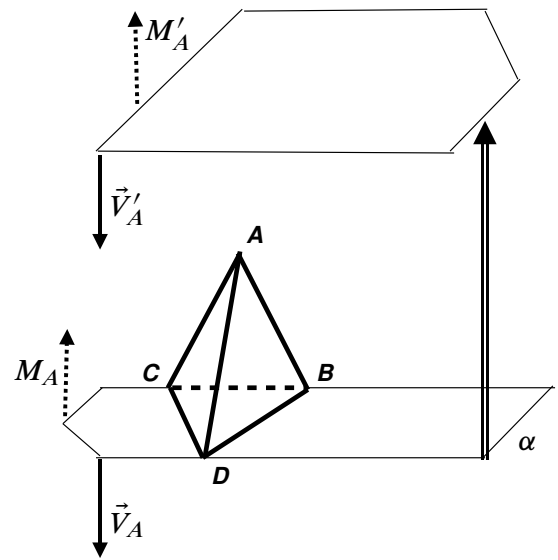

Фиг. 4

Рис. 8.

невырожденный, так как на него похож набор $T_{k+1}$. В силу индуктивного предположения это означает, что пересечение собственных полупространств н-граней набора $T_{k}$ есть правильный тетраэдр. Набор $T_{k+1}$ получается из набора $T_{k}$ заменой некоторой его н-грани $\{\alpha, \vec{F}\}$ на ее смещение, то есть на н-грань $\{\beta, \vec{H}\}$, где $F=H$. Отсюда получаем, что утверждение верно для цепочки (1) длины $k+1$.

Обратим внимание на ряд фактов, касающихся звезд.

Лемма 7. Рассмотрим звезду $\{A ; B\}$ и н-грани $\left\{\alpha ; \vec{V}_{A}\right\} \in A$ и $\left\{\beta ; \vec{V}_{B}\right\} \in B$. Если векторы $\vec{V}_{A}$ и $\vec{V}_{B}$ не коллинеарнь,, то угол между ними равен $\arccos (1 / 3)$.

Доказательство. Лемма доказывается индукцией по длине цепочки (2) с использованием леммы 4 и определений 16, 15, 5, 3 . 


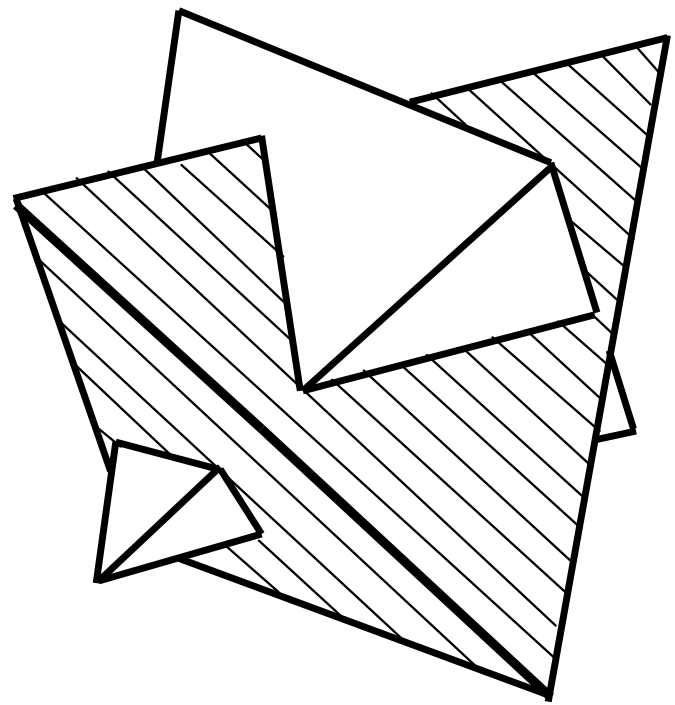

Рис. 9.

Лемма 8. Если наборы н-граней $A$ и $B$ образуют звезду, то для каждой н-грани $\left\{\alpha ; \vec{V}_{A}\right\} \in A$ найдется ровно одна н-грань $\left\{\beta ; \vec{V}_{B}\right\} \in B$ такая, что $\vec{V}_{A}=c \vec{V}_{B}$, где с $<0$.

Доказательство. Лемма доказывается индукцией по длине цепочки (2) с использованием леммы 4, первых пунктов определений 15, 5 и второго пункта определения 16.

Лемма 9. Если $\{A ; B\}-$ звезда, то $A$ и $B$ - тетраэдрические наборы.

Доказательство. Лемма доказывается индукцией по длине цепочки (2) с использованием замечания 6 и определения 14, второго пункта определения 16 и первого пункта определения 15.

Замечание 8. Пусть $\{A ; B\}$ - звезда . Из леммы 6 , следует, что для любого набора $A$ или $B$ есть две возможности: либо он является порождающим набором правильного тетраэдра, либо пересечение собственных полупространств его н-граней есть точка, являющаяся пересечением плоскостей, входящих в эти н-грани. Если каждый из наборов $A$ и $B$ является порождающим набором правильного тетраэдра, то в типичном случае эти тетраэдры расположены друг относительно друга так, как изображено на рис.9, где один из тетраэдров для наглядности заштрихован. Фигура, состоящая из двух расположенных так тетраэдров, напоминает звезду, что и объясняет выбор названия.

Лемма 10. Любой октаэдрический набор $H$ н-граней можно разбить на два поднабора А и В, образующих звезду.

Доказательство. Рассмотрим цепочку (1). Для правильного октаэдра $E$ с порождающим набором $T_{1}$ легко построить куб $Q$, центры граней которого совпадают с вершинами данного октаэдра (то есть куб, в котором правильный октаэдр расположен так, как показано на фиг. 1 рис. 6). Пусть $D$ и $F$ - тетраэдры, индуцированные кубом $Q$. Обозначим через $\alpha_{1}, \ldots, \alpha_{4}$ плоскости граней тетраэдра $D$, а через $\beta_{1}, \ldots, \beta_{4}$ плоскости граней 
тетраэдра $F$. Так как $E=D \cap F$, набор $T_{1}$ имеет вид $T_{1}=\left\{\alpha_{1} ; \vec{M}_{1}\right\}, \ldots,\left\{\alpha_{4} ; \vec{M}_{4}\right\}$, $\left\{\beta_{1} ; \vec{N}_{1}\right\}, \ldots,\left\{\beta_{4} ; \vec{N}_{4}\right\}$. Поскольку $E=D \cap F$, тетраэдры $D$ и $F$ лежат по те же стороны соответственно плоскостей $\alpha_{1}, \ldots, \alpha_{4}$ и $\beta_{1}, \ldots, \beta_{4}$, что и октаэдр $E$. Векторы $\vec{M}_{1}, \ldots, \vec{M}_{4}$, $\vec{N}_{1}, \ldots, \vec{N}_{4}-$ внешние нормали октаэдра $E$, поэтому $\vec{M}_{1}, \ldots, \vec{M}_{4}-$ внешние нормали тетраэдра $D$, а $\vec{N}_{1}, \ldots, \vec{N}_{4}-$ внешние нормали тетраэдра $F$. Но в соответствии с определением 2 это означает, что набор н-граней $\left\{\alpha_{1} ; \vec{M}_{1}\right\}, \ldots,\left\{\alpha_{4} ; \vec{M}_{4}\right\}$ является порождающим набором тетраэдра $D$, а набор н-граней $\left\{\beta_{1} ; \vec{N}_{1}\right\}, \ldots,\left\{\beta_{4} ; \vec{N}_{4}\right\}-$ порождающим набором тетраэдра $F$. Получаем, что доказываемое утверждение верно, когда длина цепочки (1) равна 1.

Предположим, что доказываемое утверждение верно, если длина цепочки (1) равна $k$, то есть набор $T_{k}$ состоит из образующих звезду поднаборов $U=\left\{\gamma_{1} ; \vec{V}_{1}\right\}, \ldots,\left\{\gamma_{4} ; \vec{V}_{4}\right\}$ и $S=\left\{\delta_{1} ; \vec{W}_{1}\right\}, \ldots,\left\{\delta_{4} ; \vec{W}_{4}\right\}$. При переходе от $T_{k}$ к $T_{k+1}$ заменяется только одна н-грань из объединения наборов $U$ и $S$. Не нарушая общности, можно считать, что н-грань $\left\{\gamma_{1} ; \vec{V}_{1}\right\}$ заменяется н-гранью $\{\varepsilon ; \vec{C}\}$. Тогда при переходе от $T_{k}$ к $T_{k+1}$ набор $U$ заменяется набором $R=\left(U \backslash\left\{\gamma_{1} ; \vec{V}_{1}\right\}\right) \cup\{\varepsilon ; \vec{C}\}$. Набор $T_{k+1}$ похож на набор $T_{k}$. Следовательно, набор $T_{k}$ невырожденный, причем пересечение собственных полупространств его н-граней имеет общую точку с пересечением собственных полупространств н-граней из набора $T_{k+1}$. Поскольку набор $U-$ часть набора $T_{k}$, а набор $R-$ часть набора $T_{k+1}$, видим, что набор $U$ невырожден, причем пересечение собственных полупространств его н-граней имеет общую точку с пересечением собственных полупространств н-граней из набора $R$. Отсюда по предположению индукции и определению звезды получаем, что пара $R$ и $S$ образует звезду, то есть, когда длина цепочки (1) равна $k+1$, набор $T_{k+1}$ разбивается на два поднабора, образующих звезду. Индукция завершена и лемма доказана.

Лемма 11. Если октаэдрический набор $H$ можно разбить на два поднабора $A$ u $B$, образующих звезду, то сделать это можно единственным образом.

Доказательство. Из лемм 9 и 6 следует, что ни набор $A$, ни набор $B$ не может содержать двух н-граней $\{\alpha ; \vec{M}\}$ и $\{\beta ; \vec{N}\}$, удовлетворяющих условию $\vec{M}=c \vec{N}$, где $c \neq 0$. Сопоставляя это с леммой 8 , получаем требуемое утверждение.

В определении 9 указано, как евклидово преобразование и гомотетия действуют на регулярные наборы н-граней. Распространим действие этих преобразований на звезды.

Определение 17. Пусть $S=\{A ; B\}-$ звезда, а $E$ - евклидово преобразование или гомотетия. Образом $E(S)$ звезды $S$ при преобразовании $E$ назовем пару $\{E(A) ; E(B)\}$.

Следующая лемма показывает, что евклидовы преобразования и гомотетии переводят множество звезд в себя.

Лемма 12. Если $S$ - звезда, а $E$ - евклидово преобразование или гомотетия, то $E(S)$ также является звездой.

Доказательство. Если длина цепочки (2) равна 1, то доказываемое утверждение вытекает из того, что поворот, параллельный перенос и гомотетия переводят тетраэдры, индуцированные одним и тем же кубом, в тетраэдры, индуцированные одним и тем же кубом. Предположим что $E(S)$ - звезда, когда длина цепочки (2) равна $k$, и покажем, что это верно, когда длина цепочки (2) равна $k+1$. Пара $\left\{C_{k+1} ; D_{k+1}\right\}$ похожа на пару $\left\{C_{k} ; D_{k}\right\}$. В силу леммы 5 это означает, что пара $\left\{E\left(C_{k+1}\right) ; E\left(D_{k+1}\right)\right\}$ похожа на пару $\left\{E\left(C_{k}\right) ; E\left(D_{k}\right)\right\}$. Поскольку по предположению пара $\left\{E\left(C_{k}\right) ; E\left(D_{k}\right)\right\}$ есть звезда, получаем требуемое утверждение. 
Для регулярных наборов было введено понятие параллельного вложения (определения 10 и 11). Распространим это понятие на звезды.

Определение 18. Пусть $A=\{M ; H\}-$ звезда. Скажем, что звезда $B$ параллельно вложена в звезду $A$, если один набор из $B$, параллельно вложен в $M$, а другой - в $H$.

Определение 19. Звезда $B$ параллельно вкладывается в звезду $A$, если существует комбинация поворота и параллельного переноса, переводящая $B$ в такую звезду, которая параллельно вложена в звезду $A$.

Сопоставляя эти определения с определениями 10 и 11, получаем следующий факт.

Лемма 13. Пусть $H_{1}$ и $H_{2}$ - два октаэдрических набора н-граней, а поднаборы $A_{t} u$ $B_{t}$ из набора $H_{t}$ составляют звезду $S_{t}, t=1,2$. Набор $H_{1}$ параллельно вкладывается 6 набор $\mathrm{H}_{2}$ тогда и только тогда, когда звезда $S_{1}$ параллельно вкладывается в звезду $S_{2}$.

Объединяя данную лемму с леммами 10 и 11, получаем следующую теорему.

Теорема 1. Пусть $H_{1}$ и $H_{2}-$ два октаэдрических набора н-граней. Тогда для набора $H_{t}$ существует единственное разбиение на поднаборы $A_{t} u B_{t}$, образующие звезду, $t=1,2$. При этом набор $\mathrm{H}_{1}$ параллельно вкладывается в набор $\mathrm{H}_{2}$ тогда и только тогда, когда звезда $\left\{A_{1}, B_{1}\right\}$ параллельно вкладывается в звезду $\left\{A_{2}, B_{2}\right\}$.

Напомним, что цель работы - нахождение критерия, определяющего возможность параллельного вложения октаэдрического многогранника или его зеркального отражения в другой октаэдрический многогранник. В силу определений 8 и 12 параллельное вложение октаэдрических многогранников можно рассматривать как частный случай параллельного вложения октаэдрических наборов н-граней. Согласно теореме 1, параллельные вложения октаэдрических наборов н-граней можно рассматривать, как частный случай параллельного вложения звезд. Поэтому для реализации нашей цели достаточно построить критерий, определяющий возможность параллельного вложения звезды или ее зеркального отражения в другую звезду. Приступим к построению такого критерия.

Учитывая лемму 6, введем понятие высоты тетраэдрического набора.

Определение 20. Высотой тетраэдрического набора $A$ назовем число $h$, определяемое по правилу:

(1) если $A$ - порождающий набор правильного тетраэдра, то полагаем $h$ равным высоте этого тетраэдра;

(2) в противном случае полагаем $h$ равным 0.

Сопоставим каждой звезде шестимерный вектор, который назовем ее профилем.

Определение 21. Пусть $\{A, B\}-$ звезда, $L_{1}, L_{2}$ - выписанные в порядке неубывания высоты тетраэдрических наборов $A$ и $B$, а $l_{1}, \ldots, l_{4}$ - выписанные в порядке неубывания расстояния между парами плоскостей из н-граней $\left\{\alpha ; \vec{V}_{A}\right\} \in A$ и $\left\{\beta ; \vec{V}_{B}\right\} \in B$, связанных условием $\vec{V}_{A}=c \vec{V}_{B}$, где $c<0$. Назовем вектор $\left(l_{1}, \ldots, l_{4}, L_{1}, L_{2}\right)$ профилем звезды $\{A, B\}$.

Нетрудно увидеть, что профили звезд обладают следующим свойством.

Лемма 14. При евклидовом преобразовании профиль звезды не меняется, а при гомотетии каждая координата профиля умножается на модуль коэффициента гомотетии. 
Обратим внимание еще на одно свойство профилей звезд.

Лемма 15. Если $\left(l_{1}, \ldots, l_{4}, L_{1}, L_{2}\right)$ - профиль звезды $H$, mо $_{1}+\ldots+l_{4}=L_{1}+L_{2}$.

Доказательство. Звезда $H$ состоит из тетраэдрических наборов $A$ и $B$. Пусть $P_{A}$ и $P_{B}$ - пересечения собственных полупространств н-граней, образующих эти наборы. Если $H$ - идеальная звезда, то $P_{A} \cap P_{B}$ - правильный октаэдр. Значит, имеется по крайней мере одна точка $M$, удовлетворяющая условию

$$
M \in P_{A} \cap P_{B}
$$

Если $P_{A}$ и $P_{B}-$ точки, то в силу (3) эти точки совпадают с $M$. Следовательно, все плоскости из н-граней, составляющих наборы $A$ и $B$, проходят через $M$. Это означает, что все координаты вектора $\left(l_{1}, \ldots, l_{4}, L_{1}, L_{2}\right)$ равны 0. Следовательно, в этом случае доказываемое утверждение выполняется.

Рассмотрим ситуацию, когда только одно из пересечений $P_{A}$ и $P_{B}$ является точкой. Не нарушая общности, можно считать, что точкой является $P_{B}$. В силу (3) эта точка совпадает с $M$. Тогда в силу леммы $6 P_{A}$ есть правильный тетраэдр. Поэтому первые четыре координаты вектора $\left(l_{1}, \ldots, l_{4}, L_{1}, L_{2}\right)$ представляют собой расстояния от точки $M$ правильного тетраэдра $P_{A}$ до его граней. Как известно, сумма этих расстояний равна высоте тетраэдра. Поскольку высота $P_{B}$ равна 0 , получаем, что и в данном случае доказываемое утверждение выполняется.

Пусть ни $P_{A}$, ни $P_{B}$ не являются точками. Тогда $P_{A}$ и $P_{B}-$ правильные тетраэдры. Учитывая лемму 8 , рассмотрим какие-либо две н-грани $\left\{\alpha ; \vec{V}_{A}\right\} \in A$ и $\left\{\beta ; \vec{V}_{B}\right\} \in B$, связанные условием $\vec{V}_{A}=c \vec{V}_{B}$, где $c<0$. Из (3) вытекает, что из ситуаций, перечисленных в предложении 4, для этих н-граней имеет место либо ситуация 1.2, либо ситуация 1.3. В обеих ситуациях точка $M$ принадлежит замкнутому слою пространства, ограниченному плоскостями $\alpha$ и $\beta$. Но это означает, что координата вектора $\left(l_{1}, \ldots, l_{4}, L_{1}, L_{2}\right)$, соответствующая расстоянию между плоскостями $\alpha$ и $\beta$, представляет собой сумму расстояний от точки $M$ до этих плоскостей, то есть сумму расстояний от $M$ до тех граней правильных тетраэдров $P_{A}$ и $P_{B}$, которые лежат на плоскостях $\alpha$ и $\beta$. Следовательно, $l_{1}+\ldots+l_{4}$ представляет собой сумму расстояний от точки $M$ до граней правильных тетраэдров $P_{A}$ и $P_{B}$. А сумма расстояний от точки правильного тетраэдра до его граней равна высоте тетраэдра. Поскольку в силу (3) $M$ принадлежит и $P_{A}$ и $P_{B}$, получаем доказываемое утверждение для случая, когда ни $P_{A}$, ни $P_{B}$ не являются точками.

Справедлива следующая теорема, доказываемая в разделах 2 и 3 настоящей работы.

Теорема 2. Звезда А или ее зеркальное отражение $A^{*}$ параллельно вкладывается в звезду В тогда и только тогда когда, когда профиль $\vec{L}=\left(l_{1}, \ldots, l_{4}, L_{1}, L_{2}\right)$ звезды $А$ связан с профилем $\vec{Q}=\left(q_{1}, \ldots, q_{4}, Q_{1}, Q_{2}\right)$ звезды В соотношениями $\vec{L} \leqslant \vec{Q}$.

Напомним, что в соответствии с общепринятым подходом соотношение $\vec{X} \leqslant \vec{Y}$, где $\vec{X}=\left(x_{1}, \ldots, x_{n}\right)$ и $\vec{Y}=\left(y_{1}, \ldots, y_{n}\right)$, понимается как сокращенная запись системы соотношений $x_{1} \leqslant y_{1}, \ldots, x_{n} \leqslant y_{n}$.

Данная теорема означает, что координаты профиля звезды можно рассматривать как параметры критерия, определяющего возможность параллельного вложения звезды или ее зеркального отражения в другую звезду.

Теперь уместно вернуться назад и напомнить, что мы исследуем вложения октаэдрических алмазов - трехмерных тел, имеющих форму октаэдрических многогранников. 


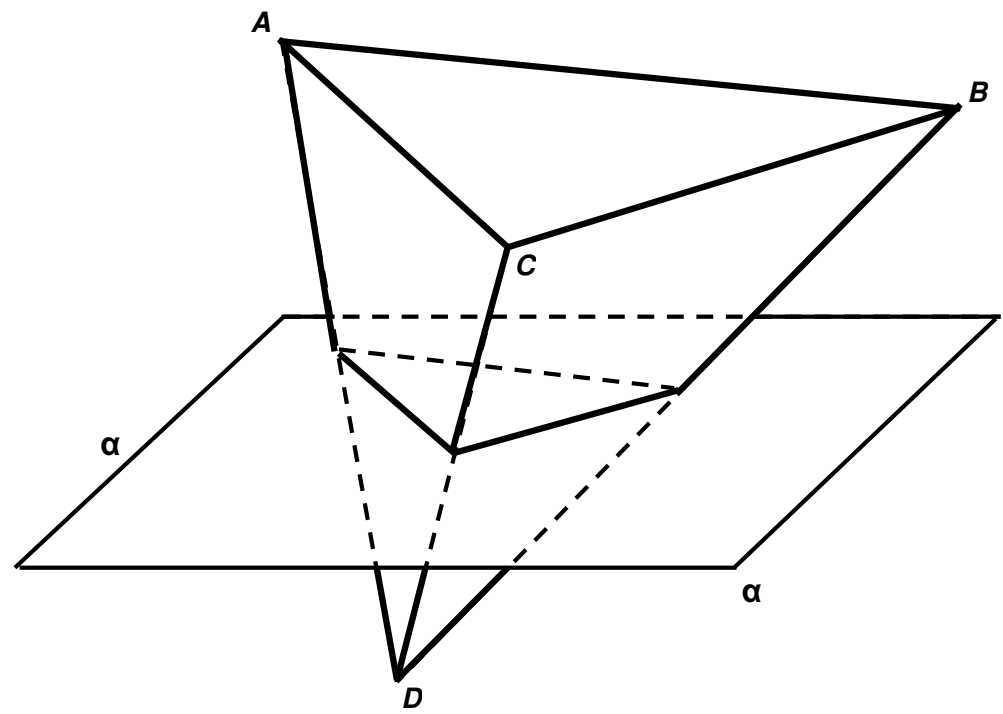

Рис. 10.

При анализе этой задачи первый шаг состоял в моделировании указанных тел с помощью более широкого класса объектов - октаэдрических наборов. При этом роль октаэдрических алмазов играли невырожденные октаэдрические наборы. Невырожденный набор $H$ моделировал кристалл, совпадающий с пересечением собственных полупространств н-граней, образующих данный набор.

Следующий шаг состоял в моделировании октаэдрических наборов посредством звезд, представляющих собой еще более широкий класс объектов. Роль невырожденных наборов н-граней (то есть роль кристаллов алмаза) играли звезды $\{A ; B\}$, у которых объединение $A \cup B$ являлось невырожденным октаэдрическим набором. Звезда $\{A ; B\}$, удовлетворяющая данному условию, моделировала кристалл алмаза, совпадающий с пересечением собственных полупространств н-граней из набора $A \cup B$.

В соответствии с этой схемой вопрос параллельного вложения октаэдрического многогранника или его зеркального отражения в другой октаэдрический многогранник, был заменен вопросом параллельного вложения звезды или ее зеркального отражения в другую звезду. В силу теоремы 2 в качестве критерия такого вложения звезд можно взять сравнение их профилей.

Во введении говорилось, что для нашей исходной задачи чрезвычайно важна возможность легко измерять (находить) параметры критерия вложимости по кристаллам алмаза. Это означает, что все координаты профиля звезды, моделирующей октаэдрический кристалл алмаза, должны легко измеряться по этому кристаллу. Перед изложением способа нахождения этих координат, обратим внимание на два известных свойства тетраэдров и одно известное свойство вложений выпуклых многогранников друг в друга.

Предложение 7. Если АВСD - тетраэдр, а $\alpha$ - плоскость, параллельная его грани $A B C$, то пересечение плоскости а с тетраэдром $A B C D$ не пусто и отлично от точки тогда и только тогда, когда грань АВC и вершина D лежат по разные стороны от плоскости $\alpha$ (см. рис. 10). 
Предложение 8. Пусть $A B C D$ и $A^{\prime} B^{\prime} C^{\prime} D^{\prime}-$ правильньле тетраэдры. Если плоскости граней $A^{\prime} B^{\prime} D^{\prime}, A^{\prime} C^{\prime} D^{\prime}, B^{\prime} C^{\prime} D^{\prime}$ тетраэдра $A^{\prime} B^{\prime} C^{\prime} D^{\prime}$ совпадают соответственно $c$ плоскостями граней $A B D, A C D, B C D$ тетраэдра $A B C D$, то

(а) вершина $D^{\prime}$ тетраэдра $A^{\prime} B^{\prime} C^{\prime} D^{\prime}$ совпадает с вершиной $D$ тетраэдра $A B C D$;

(б) грань $A^{\prime} B^{\prime} C^{\prime}$ тетраэдра $A^{\prime} B^{\prime} C^{\prime} D^{\prime}$ и грань $A B C$ тетраэдра $A B C D$ либо параллельны, либо совпадают.

Предложение 9. Если выпукльй многогранник $M$ вложен в выпукльій многогранник $T$ так, что грань $G_{M}$ многогранника $M$ лежит на грани $G_{T}$ многогранника $T$, то внешняя нормаль грани $G_{M}$ является одновременно внешней нормалью грани $G_{T}$.

Отметим также ряд фактов, касающихся звезд.

Лемма 16. Пусть $\{A ; B\}-$ звезда, и $A \cup B-$ невырожденный октаэдрический набор. Тогда для плоскости $\alpha$, входящей в какую-либо н-грань набора $A \cup B$, среди плоскостей, входящих в н-грани этого набора, найдется точно одна плоскость $\beta$, параллельная $\alpha$. При этом плоскости $\alpha$ и $\beta$ будут входить в н-грани $\{\alpha ; \vec{V}\}$ u $\{\beta ; \vec{W}\}$, удовлетворяющие условиям

(a) $\{\alpha ; \vec{V}\}$ входит в один из наборов $A$ и $B, a\{\beta ; \vec{W}\}-$ в другой;

(б) $\vec{V}=c \vec{W}$, где $c<0$.

Доказательство. Не нарушая общности, можно считать, что $\{\alpha ; \vec{V}\} \in A$. Из леммы 6 вытекает, что ни в одну н-грань из набора $A$ не может входить плоскость, параллельная плоскости $\alpha$. С другой стороны, в силу леммы 8, в наборе $B$ существует точно одна н-грань $\{\beta ; \vec{W}\}$, удовлетворяющая условию $\vec{V}=c \vec{W}$, где $c<0$. Из невырожденности набора $A \cup B$ (определение 4) вытекает, что для н-граней $\{\alpha ; \vec{V}\}$ и $\{\beta ; \vec{W}\}$ может выполняться только ситуация 2 из предложения 4. Поэтому $\alpha \neq \beta$. В силу $\vec{V}=c \vec{W}$ это означает, что $\alpha \| \beta$.

Лемма 17. Пусть звезда $\{A ; B\}$ моделирует октаэдрический кристалл алмаза $M$ (то есть $A \cup B-$ невырожденный октаэдрический набор, пересечение собственных полупространств н-граней которого совпадает с М). Тогда

(а) $A$ и В являются порождающими наборами правильных тетраэдров $A^{*} u B^{*}$;

(б) четыре грани восьмигранника $M$ лежат на гранях правильного тетраэдра $A^{*}, a$ четыре - на гранях правильного тетраэдра $B^{*}$.

Доказательство. Набор $A \cup B$ невырожденный. В силу определения 4 это означает, что $M$ - трехмерный восьмигранник, то есть имеющий внутренние точки. Совместно с леммами 6 и 9 это доказывает первое утверждение леммы. Из предложения 1 вытекает, что $M=A^{*} \cap B^{*}$. Поскольку каждый из тетраэдров $A^{*}$ и $B^{*}$ имеет по четыре грани, а $M$ имеет восемь граней, получаем второе утверждение леммы.

Лемма 18. Пусть октаэдрический многогранник $M$, моделируемый звездой $\{A ; B\}$, вложен в правильный тетраэдр $T$ так, что три грани $G_{1, M}, G_{2, M}, G_{3, M}$ многогранника $M$ лежат на трех гранях $G_{1, T}, G_{2, T}, G_{3, T}$ тетраэдра $T$. Тогда один из тетраэдрических наборов А или В является порождающим набором правильного тетраэдра $E$ такого, что грани $G_{1, M}, G_{2, M}, G_{3, M}$ многогранника $M$ лежат на гранях тетраэдра $E$. 


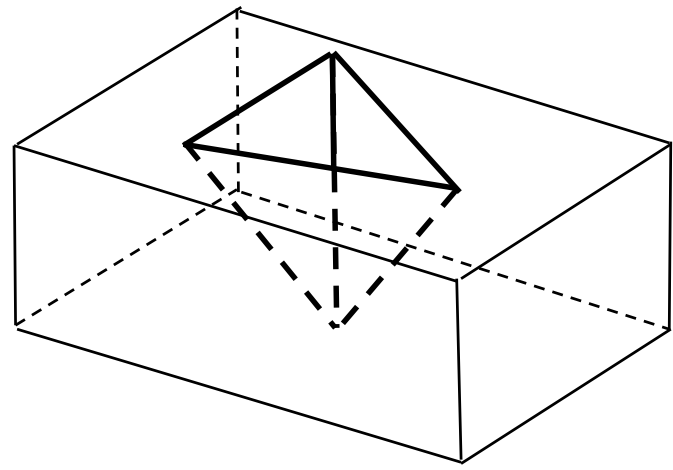

Рис. 11.

Доказательство. В силу леммы $17 A$ и $B$ - порождающие наборы правильных тетраэдров $A^{*}$ и $B^{*}$. Как известно, угол между внешними нормалями правильного тетраэдра равен $180^{\circ}-\arccos (1 / 3)$. В силу предложения 9 это означает, что внешние нормали граней $G_{1, M}, G_{2, M}, G_{3, M}$ образуют друг с другом углы равные $180^{\circ}-\arccos (1 / 3)$. С учетом предложения 2 и леммы 7 это означает, что грани $G_{1, M}, G_{2, M}, G_{3, M}$ лежат на плоскостях, входящих в какой-то один из наборов $A$ или $B$. Сопоставляя это с леммой 17 , видим, что набор, на плоскостях из н-граней которого лежат $G_{1, M}, G_{2, M}, G_{3, M}$ является искомым.

Теперь перейдем к вопросу измерения координат профиля звезды $\{A ; B\}$, моделирующей октаэдрический кристалл алмаза $M$.

Из лемм 16, 17 и предложения 2 вытекает, что для нахождения первых четырех координат профиля звезды $\{A ; B\}$ достаточно измерить расстояния между четырьмя парами параллельных граней многогранника $M$ (что нетрудно сделать, например, с помощью микрометра). В силу леммы 15 для нахождения последних двух координат профиля звезды $\{A ; B\}$ достаточно найти высоту одного из правильных тетраэдров $A^{*}$ или $B^{*}$, порождающими наборами которых являются соответственно наборы н-граней $A$ и $B$. Для нахождения этой высоты воспользуемся так называемым “измерительным брусом”, который представляет собой прямоугольный брусок (параллелепипед) с вырезанной в нем выемкой в виде правильного тетраэдра, одна из граней которого лежит на верхней грани бруска (рис. 11). Из леммы 17 следует, что при достаточно больших размерах выемки многогранник $M$ можно вложить в нее так, что три грани многогранника будут лежать на трех гранях выемки. В соответствии с леммой 18 это означает, что три грани одного из тетраэдров $A^{*}$ или $B^{*}$ лежат на тех же плоскостях, на которых лежат грани выемки, инцидентные ее вершине, расположенной внутри бруса. Не нарушая общности, будем считать, что таким тетраэдром является $A^{*}$. Обозначим вершину выемки, расположенную внутри бруса, через $H$. Из предложения 8 вытекает, что для нахождения высоты тетраэдра $A^{*}$ достаточно измерить расстояние от вершины $H$ до грани $G$ тетраэдра $A^{*}$ параллельной верхней поверхности бруса. В соответствии с леммой 17 , на грани $G$ лежит грань многогранника $M$. Следовательно, измерение высоты тетраэдра $A^{*}$ сводится к измерению расстояния от вершины $H$ до грани многогранника $M$ параллельной верхней поверхности бруса. Но, в силу лемм 16 и 17, таких граней у многогранника $M$ будет две. Из предложения 7 следует, что только одна из этих граней будет удовлетворять такому условию: многогранник $M$ и вершина $H$ лежат по одну сторону от плоскости, проходя- 
щей через данную грань. Из того же предложения 7 вытекает, что выбирать надо грань, удовлетворяющую указанному условию. Чтобы измерить расстояние от выбранной грани до вершины $H$ достаточно измерить ее расстояние до верхней поверхности бруса, что можно сделать, например, с помощью микрометра.

Приведенный способ нахождения профиля звезды, моделирующей октаэдрический алмаз, прост и поддается автоматизации. Осталось доказать теорему 2.

\section{3. Ориентированные звезды}

Если совокупность преобразований на множестве геометрических объектов включает в себя зеркальное отражение, то их анализ, как правило, становится более наглядным, если на геометрических объектах этого множества задать ориентацию. Поэтому далее мы перейдем от неориентированных звезд к ориентированным.

Вводя на звездах ориентацию, будем использовать терминологию, связанную с реперами (упорядоченными тройками линейно независимых векторов, выходящих из одной точки, называемой начальной точкой репера).

Определение 22. Репер назовем правоориентированным, если обход концов его векторов в соответствии с их порядком при взгляде из начальной точки репера осуществляется по ходу часовой стрелки. В противном случае назовем репер левоориентированным. Если два репера правоориентированы (соответственно левоориентированы), то скажем, что ориентации этих реперов совпадают. Если ориентации реперов не совпадают, то скажем, что эти ориентации противоположны.

Как известно, реперы обладают следующими свойствами.

Предложение 10. Если $R$ - репер, a $L$ - невырожденное аффинное линейное преобразование, то $L(R)$ - репер, ориентация которого

(a) совпадает с ориентацией репера $R$ при $\operatorname{det}(L)>0$;

(б) противоположна ориентаџии репера $R$ nри $\operatorname{det}(L)<0$.

Предложение 11. Рассмотрим репер $\left\langle\vec{A}_{1} ; \vec{A}_{2} ; \vec{A}_{3}\right\rangle$. Если векторы $\vec{H}$ и $\vec{P}$ таковы, что при любом $t=1,2,3$ угол между $\vec{A}_{t} u \vec{H}$ равен углу между $\overrightarrow{A_{t}} u \vec{P}$, то $\vec{H}=c \vec{P}$, где $c>0$.

Обратим внимание также на следующий факт о тетраэдрических наборах.

Лемма 19. Если $\left\{\gamma_{1} ; \vec{V}_{1}\right\}, \ldots,\left\{\gamma_{4} ; \vec{V}_{4}\right\}$ - тетраэдрический набор н-граней, то угол между векторами $\vec{V}_{t}$ и $\vec{V}_{\text {p }}$ равен $180^{\circ}-\arccos (1 / 3)$ при любых $t \neq p$.

Лемма доказывается индукцией по длине цепочки (1).

В разделе 2 наборы н-граней рассматривались как неупорядоченные. Для введения на множестве звезд ориентации удобно рассматривать упорядоченные наборы н-граней.

Определение 23. Пусть $U=\left\langle\left\{\gamma_{1} ; \vec{V}_{1}\right\}, \ldots,\left\{\gamma_{4} ; \vec{V}_{4}\right\}\right\rangle-$ упорядоченный набор н-граней. Назовем $t$-й н-гранью набора $U$ его н-грань $\left\{\gamma_{t} ; \vec{V}_{t}\right\}, t=1, \ldots, 4$.

Определение 24. Пусть $U=\left\langle\left\{\gamma_{1} ; \vec{V}_{1}\right\}, \ldots,\left\{\gamma_{4} ; \vec{V}_{4}\right\}\right\rangle$ - упорядоченный тетраэдрический набор н-граней. Скажем, что репер $\left\langle\vec{A}_{1} ; \vec{A}_{2} ; \vec{A}_{3}\right\rangle$ согласован с упорядоченным набором $U$, если $\vec{A}_{t}=c_{t} \vec{V}_{t}$, где $c_{t}>0, t=1,2,3$. 
Из предложения 10 следует, что все реперы, согласованные с каким-либо упорядоченным тетраэдрическим набором, имеют единую ориентацию (все либо левоориентированы, либо правоориентированы). Это позволяет принять ориентацию репера, согласованного с упорядоченным тетраэдрическим набором, в качестве ориентации набора. Исходя из этого, поделим тетраэдрические наборы на правые и левые.

Определение 25. Упорядоченный тетраэдрический набор назовем левым (соответственно правым), если согласованные с этим набором реперы являются левоориентированными (соответственно правоориентированными).

В определении 9 были введены евклидовы преобразования и гомотетии на неупорядоченных наборах н-граней. Распространим действие этих преобразований на упорядоченные наборы.

Определение 26. Пусть

$$
U=\left\langle\left\{\gamma_{1} ; \vec{V}_{1}\right\}, \ldots,\left\{\gamma_{n} ; \vec{V}_{n}\right\}\right\rangle
$$

- упорядоченный набор н-граней, а $E$ - евклидово преобразование или гомотетия. Образом упорядоченного набора $U$ при преобразовании $E$ назовем упорядоченный набор н-граней

$$
E(U)=\left\langle\left\{E\left(\gamma_{1}\right) ; E\left(\vec{V}_{1}\right)\right\}, \ldots,\left\{E\left(\gamma_{n}\right) ; E\left(\vec{V}_{n}\right)\right\}\right\rangle .
$$

Распространим на упорядоченные наборы н-граней понятие параллельного вложения (определения 10 и 11).

Определение 27. Пусть $A$ и $B$ - упорядоченные наборы н-граней. Набор $A$ параллельно вложен в набор $B$, если число н-граней в наборах $A$ и $B$ совпадает, а любая н-грань $\{\mu ; \vec{F}\}$ из $A$ связана со стоящей в $B$ на том же месте н-гранью $\{\gamma ; \vec{N}\}$ условиями

(a) $\vec{F}=c \vec{N}$, где $c>0$;

(б) собственное полупространство н-грани $\{\mu ; \vec{F}\}$ содержится в собственном полупространстве н-грани $\{\gamma ; \vec{N}\}$.

Определение 28. Упорядоченный набор н-граней $A$ параллельно вкладывается в упорядоченный набор н-граней $B$, если существует такая комбинация $E$ поворота и параллельного переноса, что упорядоченный набор н-граней $E(A)$ параллельно вложен в упорядоченный набор н-граней $B$.

Из этих определений, предложения 10 и определения 25, получаем следующий факт.

Лемма 20. Если упорядоченный тетраэдрический набор А параллельно вкладьвается 8 упорядоченный тетраэдрический набор $B$, то оба набора либо левые, либо правые.

Обратим внимание еще на одно свойство параллельных вложений.

Лемма 21. Пусть $E$ - евклидово преобразование. Если упорядоченный набор $A$ н-граней параллельно вложен в упорядоченный набор $B$ н-граней, то упорядоченный набор $E(A)$ н-граней параллельно вложен в упорядоченный набор $E(B)$ н-граней.

Введем, опираясь на леммы 8 и 9, понятие ориентированной звезды. 
Определение 29. Ориентированной звездой (орзвездой) назовем пару упорядоченных тетраэдрических наборов $A=\left\langle\left\{\alpha_{1} ; \vec{V}_{1}\right\}, \ldots,\left\{\alpha_{4} ; \vec{V}_{4}\right\}\right\rangle$ и $B=\left\langle\left\{\beta_{1} ; \vec{W}_{1}\right\}, \ldots,\left\{\beta_{4} ; \vec{W}_{4}\right\}\right\rangle$, удовлетворяющих следующим условиям:

(a) наборы $\left\{\alpha_{1} ; \vec{V}_{1}\right\}, \ldots,\left\{\alpha_{4} ; \vec{V}_{4}\right\}$ и $\left\{\beta_{1} ; \vec{W}_{1}\right\}, \ldots,\left\{\beta_{4} ; \vec{W}_{4}\right\}$, рассматриваемые как неупорядоченные, образуют звезду;

(б) $t$-я грань $\left\{\alpha_{t} ; \vec{V}_{t}\right\}$ упорядоченного набора $A$ и $t$-я грань $\left\{\beta_{t} ; \vec{W}_{t}\right\}$ упорядоченного набора $B$ связаны соотношением $V_{t}=c \vec{W}_{t}$, где $c<0, t=1, \ldots, 4$.

Детерминант линейного преобразования, при котором 3 вектора репера умножаются на отрицательные числа, отрицателен. Отсюда, по предложению 10, получаем, что верно следующее утверждение.

Лемма 22. В орзвезде $\{A, B\}$ один из ее наборов левый, а другой правый.

Определение 30. Тот из составляющих орзвезду $S$ упорядоченных наборов, который является левым (соответственно правым), назовем левой компонентой (соответственно правой компонентой) этой звезды.

Опираясь на определение 26, распространим действие евклидовых преобразований и гомотетий на орзвезды.

Определение 31. Пусть $E$ - евклидово преобразование или гомотетия, а $S=\{A, B\}-$ орзвезда. Образом $E(S)$ этой орзвезды при преобразовании $E$ назовем пару упорядоченных наборов $\{E(A) ; E(B)\}$.

Можно показать, что имеет место такой факт.

Лемма 23. Если $S$ - орзвезда, а $E$ - евклидово преобразование или гомотетия, то $E(S)$ - также орзвезда, причем

(a) $п р и \operatorname{det}(E)>0$ прообразом левой (соответственно правой) компоненть орзвезды $E(S)$ является левая (соответственно правая) компонента орзвезды $S$;

(б) $п р и \operatorname{det}(E)<0$ прообразом левой (соответственно правой) компоненты орзвезды $E(S)$ является правая (соответственно левая) компонента орзвезды $S$.

Эта лемма позволяет распространить понятие параллельного вложения на орзвезды.

Определение 32. Орзвезда $W$ параллельно вложена в орзвезду $S$, если левая и правая компоненты $W$ параллельно вложены соответственно в левую и правую компоненты $S$.

Определение 33. Орзвезда $W$ параллельно вкладывается в орзвезду $S$, если существует комбинация поворота и параллельного переноса, переводящая $W$ в орзвезду, параллельно вложенную в $S$.

Обратим внимание на связи между параллельными вложениями ориентированных и неориентированных звезд.

Лемма 24. Пусть $P_{1}$ и $P_{2}$ - неориентированные звезды, причем $P_{t}$ состоит из тетраэдрических наборов $A_{t} u B_{t}, t=1,2$. Если $P_{1}$ параллельно вложена в $P_{2}$, то наборы $A_{1}$, $B_{1}$ и наборы $A_{2}, B_{2}$, можно упорядочить так, что получившиеся пары упорядоченных тетраэдрических наборов $\left\{\left\langle A_{1}\right\rangle ;\left\langle B_{1}\right\rangle\right\}$ u $\left\{\left\langle A_{2}\right\rangle ;\left\langle B_{2}\right\rangle\right\}$ будут образовывать орзвезды $S_{1} u$ $S_{2}$, причем $S_{1}$ будет параллельно вложена в $S_{2}$. 
Доказательство. Не нарушая общности, будем считать, что набор $A_{1}$ параллельно вложен в $A_{2}$, а набор $B_{1}-$ в $B_{2}$. Упорядочив произвольно $A_{2}$, получим упорядоченный набор $\left\langle A_{2}\right\rangle$. В силу леммы 8, набор $B_{2}$ можно упорядочить так, что $t$-я грань $\left\{\beta_{t, 2} ; \vec{W}_{t, 2}\right\}$ упорядоченного набора $\left\langle B_{2}\right\rangle$ будет связана с $t$-й гранью $\left\{\alpha_{t, 2} ; \vec{V}_{t, 2}\right\}$ упорядоченного набора $\left\langle A_{2}\right\rangle$ соотношением

$$
\vec{V}_{t, 2}=c_{t} \vec{W}_{t, 2}, \quad \text { где } \quad c_{t}<0, \quad t=1, \ldots, 4 .
$$

Сопоставляя определения 10 и 18 с тем, что наборы $A_{1}$ и $B_{1}$ параллельно вложены соответственно в наборы $A_{2}$ и $B_{2}$, получаем, что $A_{1}$ и $B_{1}$ можно упорядочить так, что $t$-е грани $\left\{\alpha_{t, 1} ; \vec{V}_{t, 1}\right\}$ и $\left\{\beta_{t, 1} ; \vec{W}_{t, 1}\right\}$ упорядоченных наборов $\left\langle A_{1}\right\rangle$ и $\left\langle B_{1}\right\rangle$ будут связаны с $t$-ми гранями $\left\{\alpha_{t, 2} ; \vec{V}_{t, 2}\right\}$ и $\left\{\beta_{t, 2} ; \vec{W}_{t, 2}\right\}$ упорядоченных наборов $\left\langle A_{2}\right\rangle$ и $\left\langle B_{2}\right\rangle$ соотношениями

$$
\begin{aligned}
& \vec{V}_{t, 1}=q_{t} \vec{V}_{t, 2}, \quad \text { где } \quad q_{t}>0, \quad t=1, \ldots, 4, \\
& \vec{W}_{t, 1}=d_{t} \vec{W}_{t, 2}, \quad \text { где } \quad d_{t}>0, \quad t=1, \ldots, 4 \text {. }
\end{aligned}
$$

Поскольку наборы $A_{2}$ и $B_{2}$ образуют неориентированную звезду, из (4) вытекает, что наборы $\left\langle A_{2}\right\rangle$ и $\left\langle B_{2}\right\rangle$ образуют орзвезду $S_{2}$. Не нарушая общности, можно считать, что $\left\langle A_{2}\right\rangle$ и $\left\langle B_{2}\right\rangle$ являются соответственно левой и правой компонентами орзвезды $S_{2}$. Сопоставляя (5), (6) с тем, что неупорядоченные наборы $A_{1}$ и $B_{1}$ образуют звезду, видим, что наборы $\left\langle A_{1}\right\rangle$ и $\left\langle B_{1}\right\rangle$ образуют орзвезду $S_{1}$. При этом из $(5),(6)$ и того, что $\left\langle A_{2}\right\rangle$ и $\left\langle B_{2}\right\rangle$ являются соответственно левой и правой компонентами орзвезды $S_{2}$, следует, что $\left\langle A_{1}\right\rangle$ и $\left\langle B_{1}\right\rangle$ являются соответственно левой и правой компонентами орзвезды $S_{1}$. Поскольку неориентированная звезда $P_{1}$ параллельно вложена в неориентированную звезду $P_{2}$ так, что неупорядоченный набор $A_{1}$ параллельно вложен в неупорядоченный набор $A_{2}$, а неупорядоченный набор $B_{1}-$ в неупорядоченный набор $B_{2}$, получаем, что орзвезда $S_{1}$ параллельно вложена в орзвезду $S_{2}$.

Отправляясь от данной леммы, можно установить еще один факт.

Лемма 25. Пусть $P_{1}$ и $P_{2}$ - неориентированные звездыл. Если $P_{1}$ параллельно вкладывается в $P_{2}$, то наборы н-граней звезд $P_{1}$ и $P_{2}$ можно упорядочить так, что $P_{1} u P_{2}$ превратятся в орзвезды $S_{1}$ и $S_{2}$, причем $S_{1}$ будет параллельно вкладьваться в $S_{2}$.

В разделе 2 неориентированной звезде был сопоставлен вектор - ее профиль. Аналогом этого понятия для орзвезды будет вектор, называемый ее характеристикой.

Определение 34. Пусть $P$ - орзвезда, а упорядоченные тетраэдрические наборы н-граней $A_{L}=\left\langle\left\{\alpha_{1, L} ; \vec{V}_{1, L}\right\}, \ldots,\left\{\alpha_{4, L} ; \vec{V}_{4, L}\right\}\right\rangle$ и $A_{R}=\left\langle\left\{\alpha_{1, R} ; \vec{V}_{1, R}\right\}, \ldots,\left\{\alpha_{4, R} ; \vec{V}_{4, R}\right\}\right\rangle-$ eе левая и правая компоненты. Пусть $L$ и $R$ - высоты наборов $A_{L}$ и $A_{R}$, рассматриваемых как неупорядоченные, а $q_{t}$ - расстояние между плоскостями $\alpha_{t, L}$ и $\alpha_{t, R}, t=1, \ldots, 4$. Характеристикой орзвезды $P$ назовем вектор $\chi(P)=\left(q_{1}, \ldots, q_{4}, L, R\right)$.

Замечание 9. Подчеркнем различие между профилем $\left(p_{1}, p_{2}, p_{3}, p_{4}, H_{1}, H_{2}\right)$ неориентированной звезды, образованной неупорядоченными наборами н-граней $A$ и $B$, и характеристикой $\left(q_{1}, q_{2}, q_{3}, q_{4}, L, R\right)$ орзвезды, образованной упорядоченными наборами н-граней $A_{L}$ и $A_{R}$, являющимися ее левой и правой компонентами:

- $p_{1}, p_{2}, p_{3}, p_{4}$ - это расстояния между параллельными плоскостями из н-граней, составляющих наборы $A$ и $B$, упорядоченные по возрастанию, а $H_{1}, H_{2}$ - упорядоченные по возрастанию высоты правильных тетраэдров, порождаемых наборами $A$ и $B$; 
- $q_{1}, q_{2}, q_{3}, q_{4}$ - это расстояния между параллельными плоскостями из н-граней, составляющих компоненты $A_{L}$ и $A_{R}$, упорядоченные в соответствии с порядком н-граней в этих компонентах (то есть отнюдь не по возрастанию), а $L, R$ - высоты правильных тетраэдров, первый из которых порождается левой, а второй - правой компонентами орзвезды (то есть опять-таки упорядоченные не по возрастанию).

Несложно понять, что имеет место такой факт.

Лемма 26. Пусть $S$ - орзвезда с характеристикой $\chi(S)=\left(q_{1}, \ldots, q_{4}, h_{L}, h_{R}\right)$, a $E-$ евклидово преобразование. Тогда

(a) в случае $\operatorname{det}(E)>0$ преобразование $E$ не изменяет характеристики звезды, то есть $\chi(E(S))=\left(q_{1}, \ldots, q_{4}, h_{L}, h_{R}\right)$;

(б) в случае $\operatorname{det}(E)<0$ преобразование $E$ меняет в характеристике звезды местами две последние координаты, то есть $\chi(E(S))=\left(q_{1}, \ldots, q_{4}, h_{R}, h_{L}\right)$.

Сопоставляя определение характеристики орзвезды с определением профиля неориентированной звезды (определение 21), получаем еще одно свойство характеристик.

Лемма 27. Если $P=\{A, B\}-$ неориентированная звезда, то наборы $A$ и $B$ можно упорядочить так, что полученные упорядоченные наборы $\langle A\rangle$ и $\langle B\rangle$ будут образовывать орзвезду $H$, причем первые четыре координаты ее характеристики будут совпадать $c$ первыми четырьмя координатами профиля звезды $P$.

Из лемм 26 и 27 вытекает следующий факт.

Лемма 28. Если $P=\{A, B\}-$ неориентированная звезда, то ее тетраэдрические наборы $A$ и В н-граней можно упорядочить так, что полученные упорядоченнье наборь $\langle A\rangle$ u $\langle B\rangle$ образуют орзвезду $H$, удовлетворяющую хотя бы одному из следующих условий:

(а) характеристика орзвезды H совпадает с профилем звезды P;

(б) характеристика зеркального отражения H совпадает с профилем звезды $Р$.

Замечание 10. Вообще говоря, могут выполняться сразу оба условия: это будет иметь место, в случае, когда звезда $P$ зеркально симметрична.

Из данной леммы вытекает следующее важное утверждение.

Лемма 29. Пусть $H_{1}$ и $H_{2}$ - две неориентированные звезды, а $\vec{A}_{1}$ и $\vec{A}_{2}-$ их профили. Неупорядоченные наборы н-граней звезд $H_{1}$ и $H_{2}$ можно упорядочить так, что неориентированные звезды $H_{1}$ и $H_{2}$ превратятся в орзвезды $P_{1} u P_{2}$, которые вместе со своими зеркальными отражениями $P_{1}^{*} u P_{2}^{*}$ будут удовлетворять по крайней мере одному из следуюших четырех условий:

(1) $\chi\left(P_{1}\right)=\vec{A}_{1}, \chi\left(P_{2}\right)=\vec{A}_{2}$;

(2) $\chi\left(P_{1}^{*}\right)=\vec{A}_{1}, \chi\left(P_{2}\right)=\vec{A}_{2}$;

(3) $\chi\left(P_{1}\right)=\vec{A}_{1}, \chi\left(P_{2}^{*}\right)=\vec{A}_{2}$;

(4) $\chi\left(P_{1}^{*}\right)=\vec{A}_{1}, \chi\left(P_{2}^{*}\right)=\vec{A}_{2}$.

Далее нам потребуется следующее понятие, связанное с векторами. 
Определение 35. Скажем, что подстановка $\varphi$ монотонизирует вектор $\vec{X}=\left(x_{1}, x_{2}, \ldots, x_{k}\right)$, если координаты вектора $\vec{X}_{\varphi}=\left(x_{\varphi(1)}, x_{\varphi(2)}, \ldots, x_{\varphi(k)}\right)$ удовлетворяют соотношениям $x_{\varphi(1)} \leqslant x_{\varphi(2)} \leqslant \ldots \leqslant x_{\varphi(k)}$. Вектор $\vec{X}_{\varphi}$ назовем результатом монотонизации вектора $\vec{X}$. Вектор $\vec{X}$ назовем монотонным, если $\vec{X}=\vec{X}_{\varphi}$, то есть если $x_{1} \leqslant x_{2} \leqslant \ldots \leqslant x_{k}$.

Лемма 30. Пусть $\vec{X}=\left(x_{1}, x_{2}, \ldots, x_{k}\right)$ u $\vec{Y}=\left(y_{1}, y_{2}, \ldots, y_{k}\right)$ - векторы одной размерности, а $\vec{X}^{\prime}$ и $\vec{Y}^{\prime}$ - результаты их монотонизации. Если $\vec{X} \leqslant \vec{Y}$, то $\vec{X}^{\prime} \leqslant \vec{Y}^{\prime}$

Доказательство. Пусть $\varphi-$ подстановка, монотонизирующая $\vec{X}$. Из условия $\vec{X} \leqslant \vec{Y}$ вытекает, что

$$
\left(x_{\varphi(1)}, x_{\varphi(2)}, \ldots, x_{\varphi(k)}\right) \leqslant\left(y_{\varphi(1)}, y_{\varphi(2)}, \ldots, y_{\varphi(k)}\right) .
$$

Пусть $p$ - число инверсий в векторе $\left(y_{\varphi(1)}, y_{\varphi(2)}, \ldots, y_{\varphi(k)}\right)$, то есть число таких пар $(m, n)$, что $y_{\varphi(m)}>y_{\varphi(n)}, \varphi(m)<\varphi(n)$. Докажем лемму индукцией по $p$. Если $p=0$, то подстановка $\varphi$ монотонизирует не только $\vec{X}$, но и $\vec{Y}$, то есть доказываемое утверждение следует из (7). Если $p>0$, то найдется $m$ такое, что

$$
y_{\varphi(m)}>y_{\varphi(m+1)} .
$$

Подстановка $\varphi$ монотонизирует $\vec{X}$, поэтому $x_{\varphi(m)} \leqslant x_{\varphi(m+1)}$. Совместно с (7) и (8) это дает $\left(x_{\varphi(1)}, \ldots, x_{\varphi(m)}, x_{\varphi(m+1)}, \ldots, x_{\varphi(k)}\right) \leqslant\left(y_{\varphi(1)}, \ldots, y_{\varphi(m+1)}, y_{\varphi(m)}, \ldots, y_{\varphi(k)}\right)$. Но в векторе, стоящем в данном соотношении справа, число инверсий равно $p-1$. Индукционный переход завершен и лемма доказана.

Из определений профиля и характеристики (определения 21 и 34) следует, что между характеристиками орзвезд и профилями неориентированных звезд имеется следующая взаимосвязь.

Лемма 31. Если левая и правая компоненты орзвезды P есть результаты упорядочивания наборов н-граней, составляющих неориентированную звезду $S$, то характеристика $\left(q_{1}, \ldots, q_{4}, L, R\right)$ орзвезды $P$ и профиль $\left(h_{1}, \ldots, h_{4}, H_{1}, H_{2}\right)$ неориентированной звезды $S$ связаны следуюшими соотношениями:

(а) вектор $\left(h_{1}, \ldots, h_{4}\right)$ является результатом монотонизации вектора $\left(q_{1}, \ldots, q_{4}\right)$;

(б) вектор $\left(H_{1}, H_{2}\right)$ является результатом монотонизаџии вектора $(L, R)$.

Обратим внимание еще на одну связь между характеристиками орзвезд и профилями неориентированных звезд.

Лемма 32. Пусть $P_{1}, P_{2}$ - неориентированные звезды, а $H_{1}, H_{2}$ - орзвезды. Предположим, что левая и правая компоненты звезды $H_{t}$ - результаты упорядочивания наборов н-граней, составляющих звезду $P_{t}, t=1,2$. Если $\chi\left(H_{1}\right) \leqslant \chi\left(H_{2}\right)$, то профили $\vec{\pi}_{1} u \vec{\pi}_{2}$ звезд $P_{1}$ и $P_{2}$ связаны соотночением $\vec{\pi}_{1} \leqslant \vec{\pi}_{2}$.

Доказательство. Пусть

$$
\chi\left(H_{1}\right)=\left(q_{1,1}, \ldots, q_{1,4}, L_{1}, R_{1}\right), \quad \chi\left(H_{2}\right)=\left(q_{2,1}, \ldots, q_{2,4}, L_{2}, R_{2}\right) .
$$

Соотношение $\chi\left(H_{1}\right) \leqslant \chi\left(H_{2}\right)$ эквивалентно двум соотношениям

$$
\left(q_{1,1}, \ldots, q_{1,4}\right) \leqslant\left(q_{2,1}, \ldots, q_{2,4}\right), \quad\left(L_{1}, R_{1}\right) \leqslant\left(L_{2}, R_{2}\right) .
$$

Отсюда в силу лемм 31 и 30 получаем доказываемое утверждение. 
Наша ближайшая цель - показать, что теорема 2 есть следствие следующей теоремы 3.

Теорема 3. Орзвезда $H$ параллельно вкладывается в орзвезду $P$ тогда и только тогда, когда $\chi(H) \leqslant \chi(P)$.

Перед тем, как приступать к обоснованию указанной связи теорем 2 и 3, напомним следующее известное свойство евклидовых преобразований.

Предложение 12. Если евклидовы преобразования $H$ и $P$ имеют детерминант одного знака, то существует такая комбинация $E$ поворота и параллельного переноса, что $H=E P$.

Обратим внимание также на следующую достаточно просто устанавливаемую лемму.

Лемма 33. Если $E$ - евклидово линейное преобразование и орзвезда А параллельно вкладыввется в орзвезду $B$, то $E(A)$ параллельно вкладыввается в $E(B)$.

Приступим теперь к обоснованию связи теорем 2 и 3.

Лемма 34. Пусть А и В-неориентированнье звезды, а $\vec{P}_{A}$ и $\vec{P}_{B}-$ их профили. Если имеет место теорема 3 , то из возможности параллельного вложения неориентированной звезды А в неориентированную звезду В следует соотношение $\vec{P}_{A} \leqslant \vec{P}_{B}$.

Доказательство. По лемме 25 наборы н-граней звезд $A$ и $B$ можно упорядочить так, что получатся орзвезды $H$ и $P$, причем $H$ будет параллельно вкладываться в $P$. Тогда по теореме $3 \chi(H) \leqslant \chi(P)$. Отсюда в силу леммы 32 получаем требуемое утверждение.

Сопоставляя данную лемму с леммой 14, получаем такое ее обобщение.

Лемма 35. Пусть А и В - неориентированнье звездьл, а $\vec{P}_{A}$ и $\vec{P}_{B}-u x$ профили. Если имеет место теорема 2, то из возможности параллельного вложения неориентированной звезды $A$ или ее зеркального отражения в неориентированную звезду $B$ следует соотнотение $\vec{P}_{A} \leqslant \vec{P}_{B}$.

Оказывается, что имеет место также и обратное утверждение.

Лемма 36. Пусть А и В-неориентированнье звезды, а $\vec{P}_{A}$ и $\vec{P}_{B}-$ их профили. Если справедлива теорема 3 , то из соотношения $\vec{P}_{A} \leqslant \vec{P}_{B}$ следует возможность параллельного вложения неориентированной звезды $А$ или ее зеркального отражения в неориентированную звезду В.

Доказательство. Неупорядоченные наборы н-граней, составляющие звезды $A$ и $B$, можно упорядочить так, что эти звезды превратятся в орзвезды $M_{A}$ и $M_{B}$, которые вместе со своими зеркальными отражениями $M_{A}^{*}$ и $M_{B}^{*}$ будут удовлетворять по крайней мере одному из перечисленных в лемме 29 условий.

Если выполняются первое или второе условие, то есть, $\chi\left(M_{A}\right)=\vec{P}_{A}, \chi\left(M_{B}\right)=\vec{P}_{B}$ или $\chi\left(M_{A}^{*}\right)=\vec{P}_{A}, \chi\left(M_{B}\right)=\vec{P}_{B}$, то в силу теоремы $3 M_{A}$ или $M_{A}^{*}$ параллельно вкладывается в $M_{B}$. Сопоставляя этот факт с определениями параллельного вложения орзвезд и неориентированных звезд получаем, что в этом случае неориентированная звезда $A$ или ее зеркальное отражение параллельно вкладывается в неориентированную звезду $B$.

Пусть выполняется третье условие леммы 29 , то есть, $\chi\left(M_{A}\right)=\vec{P}_{A}, \chi\left(M_{B}^{*}\right)=\vec{P}_{B}$. В силу теоремы 3 это означает, что орзвезда $M_{A}$ параллельно вкладывается в орзвезду $M_{B}^{*}$. Если $E-$ зеркальное отражение, переводящее $M_{B}$ в $M_{B}^{*}$, то согласно лемме 33 орзвезда 
$E^{-1}\left(M_{A}\right)$ параллельно вкладывается в орзвезду $E^{-1}\left(M_{B}^{*}\right)=M_{B}$. Но $E^{-1}\left(M_{A}\right)-$ это зеркальное отражение $M_{A}$. Сопоставляя это с определениями параллельного вложения орзвезд и неориентированных звезд, получаем, что в рассматриваемом случае зеркальное отражение неориентированной звезды $A$ параллельно вкладывается в неориентированную звезду $B$.

Пусть выполняется четвертое условие леммы 29 , то есть, $\chi\left(M_{A}^{*}\right)=\vec{P}_{A}, \chi\left(M_{B}^{*}\right)=\vec{P}_{B}$. Согласно теореме 3 это означает, что орзвезда $M_{A}^{*}$ параллельно вкладывается в орзвезду $M_{B}^{*}$. Если $E$ - зеркальное отражение, переводящее $M_{B}$ в $M_{B}^{*}$, то в силу леммы 33 орзвезда $E^{-1}\left(M_{A}^{*}\right)$ параллельно вкладывается в орзвезду $E^{-1}\left(M_{B}^{*}\right)=M_{B}$. Пусть $H-$ зеркальное отражение, переводящее $M_{A}$ в $M_{A}^{*}$. Тогда $E^{-1}\left(M_{A}^{*}\right)=E^{-1}\left(H\left(M_{A}\right)\right)$. Так как $E^{-1}$ и $H$ являются зеркальными отражениями, детерминант их произведения равен 1. Ввиду предложения 12 из этого вытекает, что $E^{-1} H$ представляет собой комбинацию поворота и параллельного переноса. Сопоставляя это обстоятельство с тем, что орзвезда $E^{-1}\left(H\left(M_{A}\right)\right)$ параллельно вкладывается в орзвезду $M_{B}$, и учитывая, что в силу предложения 12 произведение любого числа поворотов и параллельных переносов равно комбинации одного параллельного переноса и одного поворота, получаем, что орзвезда $M_{A}$ параллельно вкладывается в орзвезду $M_{B}$. Вместе с определениями параллельного вложения орзвезд и неориентированных звезд это означает, что в рассматриваемом случае неориентированная звезда $A$ параллельно вкладывается в неориентированную звезду $B$.

Из лемм 35 и 36 получаем, что теорема 2 следует из теоремы 3. Доказательству теоремы 3 посвящена оставшаяся часть работы.

\section{4. Доказательство теоремы 3}

Перед тем, как перейти к доказательству этой теоремы, сформулируем в удобной для нас форме известное свойство параллельных плоскостей, связанное с делением плоскостью пространства на два полупространства.

Предложение 13. Пусть $M_{A}$ и $M_{B}$ - собственные полупространства н-граней $\{\alpha ; \vec{A}\} u$ $\{\beta ; \vec{B}\}$, у которых $\vec{A}=c \vec{B}$, где $c>0$. Если $P-$ точка из $M_{A} \cap M_{B}$, а $p_{\alpha} u p_{\beta}-e е$ расстояния до плоскостей $\alpha$ и $\beta$, то $p_{\alpha} \leqslant p_{\beta}$ тогда и только тогда, когда $M_{A} \subseteq M_{B}$ (см. рис. 12, где точечныли стрелками указано, по какую сторону от плоскостей $\alpha$ и $\beta$ расположень полупространства $M_{A} u M_{B}$ ).

Определение 36. Пусть $U=\left\langle\left\{\gamma_{1} ; \vec{V}_{1}\right\}, \ldots,\left\{\gamma_{4} ; \vec{V}_{4}\right\}\right\rangle-$ упорядоченный тетраэдрический набор н-граней, а $T$ - точка из пересечения собственных полупространств н-граней набора $U$. Следом точки $T$ в наборе $U$ назовем вектор $\left(p_{1}, \ldots, p_{4}\right)$, где $p_{t}-$ расстояние от точки $T$ до плоскости $\gamma_{t}, t=1, \ldots, 4$.

Лемма 37. Пусть $A$ и $B$-упорядоченные тетраэдрические наборы н-граней, а $P_{A} и P_{B}$ - пересечения собственных полупространств н-граней этих наборов. Если упорядоченный набор $A$ параллельно вложен в упорядоченный набор $B$, то $P_{A} \cap P_{B}=P_{A} u$ для любой точки $M$ из $P_{A} \cap P_{B}$ ее след в наборе $A$ не превосходит ее следа в наборе $B$.

Доказательство. Соотношение $P_{A} \cap P_{B}=P_{A}$ вытекает из определения 27. Из этого же определения и предложения 13 получаем требуемое соотношение между следами точки $M$ в наборах $A$ и $B$. 


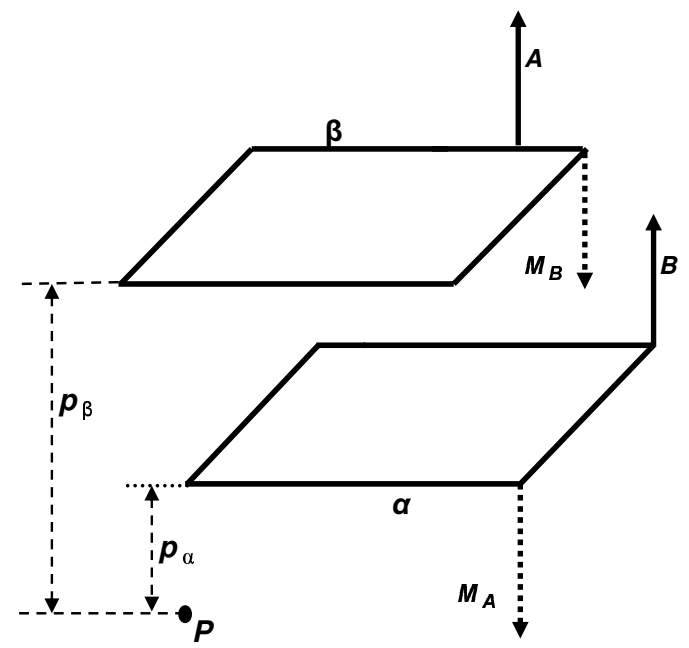

Рис. 12.

Лемма 38. Пусть $A$ и $B$-левая и правая компоненты орзвезды $H$, а $P_{A}$ и $P_{B}$ - пересечения собственных полупространств н-граней из $A$ и В. Пусть $M-$ точка из пересечения, mo ecmb,

$$
M \in P_{A} \cap P_{B}
$$

Если $\left(a_{1}, \ldots, a_{4}\right)$ и $\left(b_{1}, \ldots, b_{4}\right)$ - следы точки $M$ в наборах $A$ и $B, a\left(q_{1}, \ldots, q_{4}, L, R\right)-$ характеристика звезды $\mathrm{H}$, то

$$
\begin{aligned}
q_{t} & =a_{t}+b_{t}, & t & =1,2,3,4, \\
L & =a_{1}+\ldots+a_{4}, & R & =b_{1}+\ldots+b_{4} .
\end{aligned}
$$

Доказательство. В силу леммы 6 возможны три случая:

(a) $P_{A}$ и $P_{B}$ являются точками;

(б) только одно из пересечений $P_{A}$ и $P_{B}$ является точкой;

(в) ни $P_{A}$, ни $P_{B}$ не являются точками.

В случае (а), в силу (9), точки $P_{A}$ и $P_{B}$ должны совпасть с $M$. Следовательно, все плоскости, из н-граней наборов $A$ и $B$ проходят через $M$, и потому следы $M$ в этих наборах - нулевые векторы. Из определений 34 и 20 вытекает, что в случае (а) вектор $\chi(H)$ также является нулевым, то есть, соотношения (10) и (11) выполняются.

В случае (б), когда либо $P_{A}$ либо $P_{B}$ является точкой, не нарушая общности, будем считать, что точкой является $P_{B}$. В силу (9) эта точка совпадает с $M$. В силу леммы $6, P_{A}$ есть правильный тетраэдр. Поэтому первые четыре координаты вектора $\left(q_{1}, \ldots, q_{4}, L, R\right)-$ это расстояния от точки $M$ правильного тетраэдра $P_{A}$ до его граней. Сумма этих расстояний равна высоте правильного тетраэдра. В силу определения 20, высота $P_{B}$ равна 0 , то есть в рассматриваемом случае соотношения (10) и (11) также выполняются.

В случае (в) ни $P_{A}$, ни $P_{B}$ не являются точками, то есть, в силу леммы $6 P_{A}$ и $P_{B}$ являются правильными тетраэдрами. Поскольку, согласно (9), $M$ принадлежит обоим 


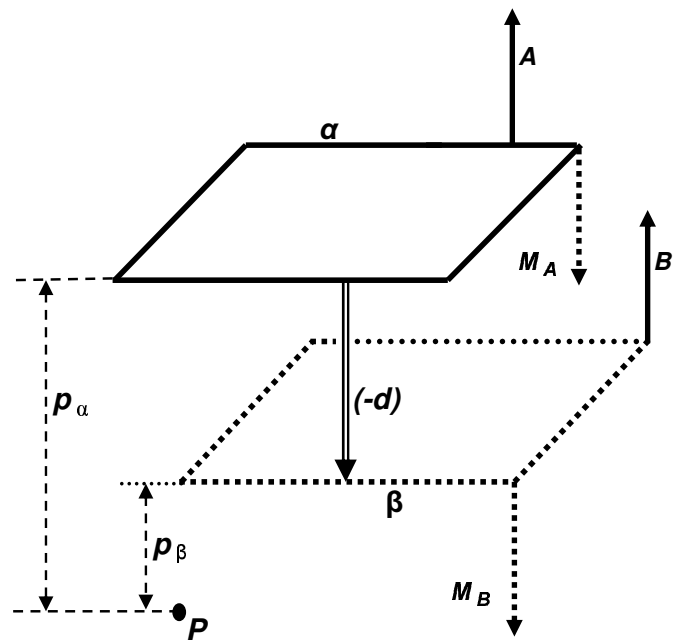

Рис. 13.

тетраэдрам, а сумма расстояний от точки правильного тетраэдра до его граней равна высоте тетраэдра, получаем, что соотношения (11) выполняются.

Перейдем к обоснованию соотношений (10). Пусть $t$-ми н-гранями в наборах $A$ и $B$ будут $\left\{\alpha_{t} ; \vec{V}_{A, t}\right\} \in A$ и $\left\{\beta_{t} ; \vec{V}_{B, t}\right\} \in B, t=1, \ldots, 4$. В силу второго пункта определения 29 , $\vec{V}_{A, t}=c_{t} \vec{V}_{B, t}$, где $c_{t}<0, t=1, \ldots, 4$. Сопоставляя это с (9), получаем, что при любом $t=1, \ldots, 4$ для н-граней $\left\{\alpha_{t} ; \vec{V}_{A, t}\right\}$ и $\left\{\beta_{t} ; \vec{V}_{B, t}\right\}$ имеет место ситуация 2 или 3 из предложения 4. В обеих ситуациях точка $M$ принадлежит замкнутому слою пространства, ограниченному плоскостями $\alpha_{t}$ и $\beta_{t}$. Следовательно, $t$-я координата вектора $\chi(H)$ есть сумма расстояний от $M$ до плоскостей $\alpha_{t}$ и $\beta_{t}, t=1, \ldots, 4$. Но это означает выполнение соотношений (10).

Лемма 39. Если орзвезда $S$ параллельно вкладьвается в орзвезду $Q$, то $\chi(S) \leqslant \chi(Q)$.

Доказательство. Существует такая комбинация $E$ поворота и параллельного переноса, что орзвезда $E(S)$ параллельно вложена в орзвезду $Q$. Пусть $L_{E, S}$ и $R_{E, S}-$ пересечения собственных полупространств н-граней, образующих левую и правую компоненты орзвезды $E(S)$, а $L_{Q}$ и $R_{Q}$ - пересечения собственных полупространств н-граней, образующих левую и правую компоненты звезды $Q$. Тогда существует точка $M \in\left(L_{E, S} \cap R_{E, S}\right) \cap\left(L_{Q} \cap R_{Q}\right)$. В силу леммы 37, следы точки $M$ в левой и правой компонентах орзвезды $E(S)$ не превосходят ее следов соответственно в левой и правой компонентах орзвезды $Q$. Согласно лемме 38 , это означает, что $\chi(E(S)) \leqslant \chi(Q)$. Из этого неравенства и леммы 26 получаем доказываемое утверждение.

Данная лемма устанавливает, что условие, накладываемое в теореме 3 на характеристики, необходимо. Оставшаяся часть работы посвящена доказательству достаточности этого условия.

Сначала сформулируем в терминах операции смещения свойства параллельных плоскостей, связанных с делением плоскостью пространства на два полупространства. 


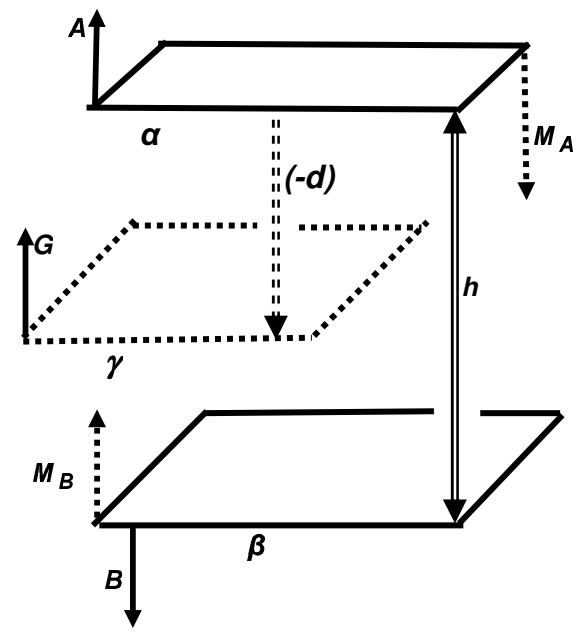

Фиг. 1

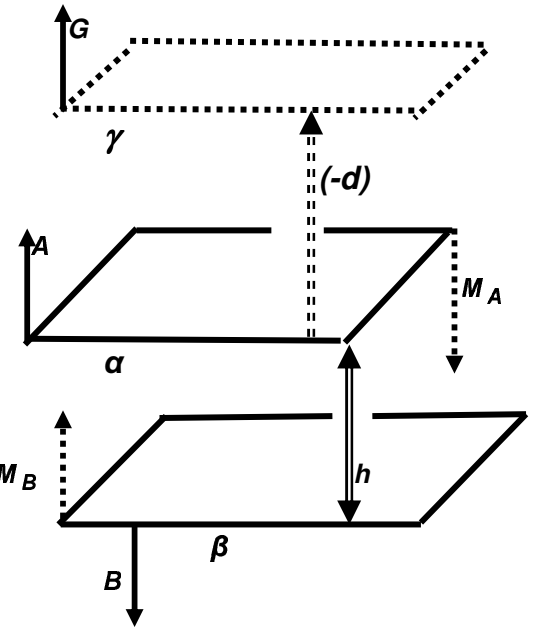

Фиг. 2

Рис. 14.

Предложение 14. Рассмотрим н-грань $\{\alpha ; \vec{A}\}$ и число $d \geqslant 0$. Если н-грань $\{\beta ; \vec{B}\}$ является $(-d)$-смещчением н-грани $\{\alpha ; \vec{A}\}$ (см. рис. 13), то

(a) собственное полупространство $M_{B}$ н-грани $\{\beta ; \vec{B}\}$ принадлежит собственному полупространству $M_{A}$ н-грани $\{\alpha ; \vec{A}\}$;

(б) для любой точки $P$ из $M_{B}$ ее расстояния $p_{\alpha}$ и $p_{\beta}$ до плоскостей $\alpha$ и $\beta$ удовлетворяют соотношению $p_{\alpha}=p_{\beta}+d$.

Предложение 15. Пусть $M_{A}$ и $M_{B}$ - собственные полупространства н-граней $\{\alpha ; \vec{A}\} u$ $\{\beta ; \vec{B}\}$, у которых $\vec{A}=c \vec{B}$, где $c<0$, а $h-$ толщина замкнутого слоя пространства, ограниченного плоскостями $\alpha$ и $\beta$. Рассмотрим какое-либо $d \leqslant h$. Если $M_{A} \cap M_{B} \neq \varnothing$ (то есть, если имеет место ситуаџия 2 или ситуаџия 3 из предложения 4), то:

(а) н-грань $\{\gamma ; \vec{G}\}$, являющяаяся $(-d)$-смещзением н-грани $\{\alpha ; \vec{A}\}$, удовлетворяет условию $\gamma \subseteq M_{B}$;

(б) расстояние между плоскостями $\alpha$ и $\beta$ равно $h-d$.

(См. рис. 14, где на фиг. 1 изображена ситуаџия $d>0$, а на фиг. 2 - ситуаџия $d<0$ ).

Опираясь на предложение 14, несложно установить следующую лемму.

Лемма 40. Пусть $A=\left\langle\left\{\gamma_{1} ; \vec{V}_{1}\right\}, \ldots,\left\{\gamma_{4} ; \vec{V}_{4}\right\}\right\rangle-$ упорядоченный тетраэдрический набор н-граней, а $h$ - высота набора $A$. Если $0 \leqslant d \leqslant h$, то при замене какой-либо н-грани из $A$ на результат ее $(-d)$-смещения получится упорядоченный тетраэдрический набор $B$, параллельно вложенный в набор $A$.

Обратим внимание еще на две несложно доказываемые леммы.

Лемма 41. Если A-тетраэдрический набор н-граней, то плоскости, входящие в любые три н-грани этого набора пересекаются в точке, принадлежащей собственному полупространству четвертой н-грани этого набора. 
Лемма 42. Пусть левая и правая компоненть орзвезды $P$ представляют собой упорядоченные тетраэдрические наборы н-граней $A_{L}=\left\langle\left\{\alpha_{1, L} ; \vec{V}_{1, L}\right\}, \ldots,\left\{\alpha_{4, L} ; \vec{V}_{4, L}\right\}\right\rangle u$ $A_{R}=\left\langle\left\{\alpha_{1, R} ; \vec{V}_{1, R}\right\}, \ldots,\left\{\alpha_{4, R} ; \vec{V}_{4, R}\right\}\right\rangle$. Тогда плоскость $\alpha_{t, R}$ лежит в собственном полупространстве $P_{t, L}$ н-грани $\left\{\alpha_{t, L} ; \vec{V}_{t, L}\right\}$, а плоскость $\alpha_{t, L}-6$ собственном полупространстве $P_{t, R}$ н-грани $\left\{\alpha_{t, R} ; \vec{V}_{t, R}\right\}, t=1, \ldots, 4$.

Учитывая лемму 41, введем следующую терминологию.

Определение 37. Пусть $A$ - упорядоченный тетраэдрический набор. Назовем $t$-й вершиной набора $A$ точку пересечения плоскостей, входящих в н-грани, стоящие в наборе $A$ на местах, отличных от $t$-го, $t=1, \ldots, 4$.

Замечание 11. Обратим внимание, что в случае, когда пересечение собственных полупространств н-граней, входящих в набор $A$, представляет собой точку, $k$-я вершина набора $A$ является одновременно и его $t$-й вершиной при всех $k, t \in\{1, \ldots, 4\}$.

Из леммы 42 вытекает следующее свойство вершин компонент орзвезд.

Лемма 43. Если $H$ - орзвезда, то $t$-я вершина ее левой (соответственно правой) компоненты принадлежит пересечению собственных полупространств н-граней из правой (соответственно левой) компоненты орзвезды $H$, стоящих в этой компоненте на месmах, отличных от $t$-го, $t=1, \ldots, 4$.

Чтобы сделать наглядней дальнейшее изложение, поступим следующим образом. Перед доказательством достаточности условия из теоремы 3 выделим ряд относительно простых фактов. Эти факты касаются того, что происходит с орзвездой, когда н-грань, принадлежащая какой-либо из компонент орзвезды, заменяется своим смещением, а величина смещения отрицательна. Сначала покажем, что при достаточно естественных ограничениях на величину смещения пересечение собственных полупространств н-граней, входящих в упорядоченные наборы, получившиеся из компонент орзвезды, не пусто.

Лемма 44. Пусть $U_{1}$ и $U_{2}$ - левая и правая компоненть орзвезды $D$ с характеристикой $\chi(D)=\left(q_{1}, \ldots, q_{4}, H_{1}, H_{2}\right)$. Выберем $d \geqslant 0, t \in\{1, \ldots, 4\} u m, n \in\{1,2\}$, где $n \neq m$. Заменив $t$-ю грань набора $U_{m}$ на ее $(-d)$-смещение, получим новый упорядоченный набор $U_{m}^{*}$. Если

$$
d \leqslant \min \left(q_{t}, H_{m}\right)
$$

то пересечение собственных полупространств н-граней набора $U_{m}^{*}$, имеет общую точку M с пересечением собственных полупространств н-граней набора $U_{n}$.

Доказательство. Не нарушая общности, можно считать, что

$$
t=1, \quad m=1, \quad n=2 .
$$

Предположим, что $U_{1}=\left\langle\left\{\alpha_{1} ; \vec{V}_{1}\right\}, \ldots,\left\{\alpha_{4} ; \vec{V}_{4}\right\}\right\rangle, U_{2}=\left\langle\left\{\beta_{1} ; \vec{W}_{1}\right\}, \ldots,\left\{\beta_{4} ; \vec{W}_{4}\right\}\right\rangle$. Обозначим собственные полупространства н- граней $\left\{\alpha_{k} ; \vec{V}_{k}\right\}$ и $\left\{\beta_{k} ; \vec{W}_{k}\right\}$ соответственно через $F_{k}$ и $G_{k}$, а $k$-е вершины упорядоченных тетраэдрических наборов $U_{1}$ и $U_{2}-$ соответственно через $Q_{k}$ и $S_{k}, k=1, \ldots, 4$. В силу леммы 43 ,

$$
Q_{1} \in G_{2} \cap G_{3} \cap G_{4}, \quad S_{1} \in F_{2} \cap F_{3} \cap F_{4} .
$$


В соответствии с определением 37 ,

$$
Q_{1} \in F_{2} \cap F_{3} \cap F_{4}, \quad S_{1} \in G_{2} \cap G_{3} \cap G_{4} .
$$

Согласно предложению 2, пересечение собственных полупространств н-граней есть выпуклое множество. Учитывая (14) и (15), получаем, что отрезок $Q_{1} S_{1}$ принадлежит пересечению

$$
Q_{1} S_{1} \subseteq\left(F_{2} \cap F_{3} \cap F_{4}\right) \cap\left(G_{2} \cap G_{3} \cap G_{4}\right)
$$

Рассмотрим н-грань $\left\{\alpha_{1}^{*} ; \vec{V}_{1}^{*}\right\}$, являющуюся $(-d)$-смещением н-грани $\left\{\alpha_{1} ; \vec{V}_{1}\right\}$. Пусть $F_{1}^{*}-$ собственное полупространство этой н-грани. Тетраэдрические наборы $U_{1}$ и $U_{2}$ образуют звезду. Поэтому $F_{1} \cap G_{1} \neq \varnothing$, что вместе с (12) и первым пунктом предложения 15 дает включение

$$
\alpha_{1}^{*} \subseteq G_{1}
$$

В силу леммы 41,

$$
Q_{1} \in F_{1}, \quad S_{1} \in G_{1} .
$$

Из (12), (13) и предложения 6 получаем, что собственное полупространство $F_{1}^{*}$ н-грани $\left\{\alpha_{1}^{*} ; \vec{V}_{1}^{*}\right\}$ удовлетворяет соотношению

$$
Q_{1} \in F_{1}^{*} \text {. }
$$

Возможны две ситуации $S_{1} \in F_{1}^{*}$ и $S_{1} \notin F_{1}^{*}$. Если $S_{1} \in F_{1}^{*}$, то, в силу (14), (15) и (18), вершину $S_{1}$ можно взять за искомую точку $M$. Пусть $S_{1} \notin F_{1}^{*}$. Тогда в силу (19) на отрезке $Q_{1} S_{1}$ должна быть точка $E \in \alpha_{1}^{*}$. Из (16) и (17) получаем, что $E \in\left(F_{1}^{*} \cap F_{2} \cap F_{3} \cap F_{4}\right) \cap\left(G_{1} \cap G_{2} \cap G_{3} \cap G_{4}\right)$. Поэтому $E$ можно принять в качестве искомой точки $M$.

Опираясь на данную лемму, установим еще один факт о том, что происходит с орзвездой, когда н-грань, принадлежащая ее компоненте, заменяется своим смещением, где величина смещения отрицательна. Оказывается, что при ограничениях на величину смещения, упомянутых в лемме 44, орзвезда переходит в орзвезду, параллельно вложенную в исходную орзвезду. При этом характеристика полученной орзвезды достаточно естественно связана с характеристикой исходной орзвезды.

Лемма 45. Пусть выполнень все условия леммь 44. Тогда упорядоченные наборь $U_{m}^{*} u$ $U_{n}$ - это левая и правая компоненты орзвезды $D^{*}$, параллельно вложенной в орзвезду $D$. При этом вектор $\chi\left(D^{*}\right)=\left(q_{1}^{*}, \ldots, q_{4}^{*}, H_{1}^{*}, H_{2}^{*}\right)$ связан с вектором $\chi(D)$ соотночениями

$$
\begin{array}{rlrl}
q_{t}^{*} & =q_{t}-d, \quad q_{k}^{*} & =q_{k} \quad \text { при } k \neq t, \\
H_{m}^{*} & =H_{m}-d, \quad H_{n}^{*}=H_{n} .
\end{array}
$$

Доказательство. Не нарушая общности, можно считать, что

$$
t=1, \quad m=1, \quad n=2 .
$$

Если $d=0$, то $U_{1}^{*}$ совпадает с $U_{1}$, то есть $D^{*}$ совпадает с $D$. Поэтому в случае $d=0$ доказываемое утверждение тривиально верно. Пусть

$$
d>0 \text {. }
$$


Предположим, что $U_{1}=\left\langle\left\{\alpha_{1} ; \vec{V}_{1}\right\}, \ldots,\left\{\alpha_{4} ; \vec{V}_{4}\right\}\right\rangle, U_{2}=\left\langle\left\{\beta_{1} ; \vec{W}_{1}\right\}, \ldots,\left\{\beta_{4} ; \vec{W}_{4}\right\}\right\rangle$. Обозначим собственные полупространства н-граней $\left\{\alpha_{k} ; \vec{V}_{k}\right\}$ и $\left\{\beta_{k} ; \vec{W}_{k}\right\}$ соответственно через $F_{k}$ и $G_{k}, k=1, \ldots, 4$. Так как $D-$ орзвезда с характеристикой $\chi(D)=\left(q_{1}, \ldots, q_{4}, H_{1}, H_{2}\right)$,

$$
F_{1} \cap \ldots \cap F_{4} \text { есть правильный тетраэдр высоты } H_{1}>0 \text {. }
$$

Следовательно,

$$
\text { набор } U_{1} \text { невырожденный. }
$$

Рассмотрим н-грань $\left\{\alpha_{1}^{*} ; \vec{V}_{1}^{*}\right\}$, являющуюся $(-d)$-смещением н-грани $\left\{\alpha_{1} ; \vec{V}_{1}\right\}$, и собственное полупространство $F_{1}^{*}$ этой н-грани. Из (12), (22), (24) вытекает, что $\left(F_{1} \cap F_{2} \cap \ldots \cap F_{4}\right)$ и $\left(F_{1}^{*} \cap F_{2} \cap \ldots \cap F_{4}\right)$ имеют общую точку. Сопоставляя это с $(25)$ и определением похожести (определение 5) видим, что если рассматривать наборы $U_{1}^{*}$ и $U_{1}$ как неупорядоченные, Tо

$$
\text { набор } U_{1}^{*} \text { похож на набор } U_{1} \text {. }
$$

Из (12), (22), предложения 6 и определения высоты тетраэдрического набора (определение 20) вытекает, что высота $H_{1}^{*}$ тетраэдрического набора $U_{1}^{*}$, рассматриваемого как неупорядоченный, удовлетворяет соотношению

$$
H_{1}^{*}=H_{1}-d
$$

Упорядоченные наборы $U_{1}$ и $U_{2}$ образуют орзвезду. Поэтому $F_{1} \cap G_{1} \neq \varnothing, V_{1}=c W_{1}$, где $c<0$. В соответствии с неравенством (12), условием (22) и предложением 15, это означает, что расстояние $q_{1}^{*}$ между плоскостями $\alpha_{1}^{*}$ и $\beta_{1}$ удовлетворяет соотношению

$$
q_{1}^{*}=q_{1}-d .
$$

Из (12), (22) и леммы 40 вытекает, что упорядоченный набор $U_{1}^{*}$ параллельно вложен в упорядоченный набор $U_{1}$. Совместно с (26) и леммой 44 это означает, что $U_{1}^{*}$ и $U_{2}$ можно рассматривать, как левую и правую компоненты орзвезды $D^{*}$, параллельно вложенной в орзвезду $D$. Сопоставляя (27) и (28) с тем, что ввиду (22) при переходе от $U_{1}$ к $U_{1}^{*}$ заменялась только первая н-грань набора $U_{1}$, получаем, что $\chi\left(D^{*}\right)=\left(q_{1}^{*}, \ldots, q_{4}^{*}, H_{1}^{*}, H_{2}^{*}\right)$ удовлетворяет условиям (20) и (21).

Эта лемма может быть естественным образом обобщена на случай, когда несколько н-граней из компонент орзвезды заменяются своими смещениями. Нам будет удобно сформулировать требуемое обобщение в виде следующей леммы.

Лемма 46. Пусть $S$ - орзвезда, а $F-$ точка из пересечения собственных полупространств н-граней, входящих в компоненты $S$. Пусть следами $F$ в левой и правой компоненте орзвезды $S$ являются соответственно $\left(a_{1}, \ldots, a_{4}\right) u\left(b_{1}, \ldots, b_{4}\right)$. Наряду с орзвездой $S$ рассмотрим орзвезду $Q$. Пусть левая и правая компоненты $Q-$ это A и B. Заменив $t$-e н-грани в А на их $\left(-a_{t}\right)$-смещеения, а t-е н-грани в $B-$ на их $\left(-b_{t}\right)$-смещения, $t=1, \ldots, 4$, получим упорядоченные наборы $A^{*}$ и $B^{*}$. Если $\chi(S) \leqslant \chi(Q)$, то $A^{*}$ и $B^{*}$ - это левая $u$ правая компоненты орзвезды $Q^{*}$, параллельно вложенной в орзвезду $Q$.

Доказательство. Пусть

$$
\chi(Q)=\left(q_{1}, \ldots, q_{4}, H_{1}, H_{2}\right) .
$$


Отправляясь от $A$ и $B$, построим две цепочки пар упорядоченных наборов н-граней

$$
\begin{aligned}
\{A, B\} & =\left\{A_{0}, B\right\} \rightarrow\left\{A_{1}, B\right\} \rightarrow\left\{A_{2}, B\right\} \rightarrow\left\{A_{3}, B\right\} \rightarrow\left\{A_{4}, B\right\}, \\
\left\{A_{4}, B\right\} & =\left\{A_{4}, B_{0}\right\} \rightarrow\left\{A_{4}, B_{1}\right\} \rightarrow\left\{A_{4}, B_{2}\right\} \rightarrow\left\{A_{4}, B_{3}\right\} \rightarrow\left\{A_{4}, B_{4}\right\}=\left\{A^{*}, B^{*}\right\},
\end{aligned}
$$

считая, что

(a) упорядоченный набор $A_{t+1}$ получается из упорядоченного набора $A_{t}$ заменой $t$-й н-грани на ее $\left(-a_{t}\right)$-смещение, $t=0,1,2,3$;

(б) упорядоченный набор $B_{t+1}$ получается из упорядоченного набора $B_{t}$ заменой $t$-й н-грани на ее $\left(-b_{t}\right)$-смещение, $t=0,1,2,3$.

Учитывая лемму 38 и соотношение $\chi(S) \leqslant \chi(Q)$, последовательно применим лемму 45 к каждому переходу в цепочках (30) и (31). В силу (29) получаем, что $A_{4}$ и $B_{4}$ представляют собой левую и правую компоненты орзвезды $Q^{*}$, параллельно вложенной в орзвезду $Q$ и удовлетворяющей условию

$$
\chi\left(Q^{*}\right)=\left(q_{1}-a_{1}-b_{1}, \ldots, q_{4}-a_{4}-b_{4}, H_{1}-\sum_{t=1}^{4} a_{t}, H_{2}-\sum_{t=1}^{4} b_{t}\right)
$$

Далее нам потребуется еще ряд понятий, связанных с реперами.

Определение 38. Реперы $\left\langle\vec{A}_{1} ; \vec{A}_{2} ; \vec{A}_{3}\right\rangle$ и $\left\langle\vec{B}_{1} ; \vec{B}_{2} ; \vec{B}_{3}\right\rangle$ назовем эквивалентными, если выполнены следующие два условия:

(a) реперы одинаково ориентированы;

(б) угол между векторами $A_{j}$ и $A_{t}$ равен углу между векторами $B_{j}$ и $B_{t}, j=1,2,3$, $t=1,2,3$.

Понятие эквивалентности реперов позволяет сформулировать в удобной форме следующее известное свойство поворотов и параллельных переносов.

Предложение 16. Эквивалентность реперов $\left\langle\vec{A}_{1} ; \vec{A}_{2} ; \vec{A}_{3}\right\rangle$ u $\left\langle\vec{B}_{1} ; \vec{B}_{2} ; \vec{B}_{3}\right\rangle$ необходима $и$ достаточна для существования комбинации ч поворота и параллельного переноса, переводящей репер $\left\langle\vec{B}_{1} ; \vec{B}_{2} ; \vec{B}_{3}\right\rangle$ в такой репер $\left\langle\varphi\left(\vec{B}_{1}\right) ; \varphi\left(\vec{B}_{2}\right) ; \varphi\left(\vec{B}_{3}\right)\right\rangle$, что

(а) начальнье точки реперов $\left\langle\vec{A}_{1} ; \vec{A}_{2} ; \vec{A}_{3}\right\rangle$ u $\left\langle\varphi\left(\vec{B}_{1}\right) ; \varphi\left(\vec{B}_{2}\right) ; \varphi\left(\vec{B}_{3}\right)\right\rangle$ cовпадают;

(б) при любом $t=1,2,3$ имеет место равенство $\varphi\left(\vec{B}_{t}\right)=c_{t} \vec{A}_{t}$, где $c_{t}>0$.

Определение 39. Пусть $U=\left\langle\left\{\gamma_{1} ; \vec{V}_{1}\right\}, \ldots,\left\{\gamma_{4} ; \vec{V}_{4}\right\}\right\rangle-$ упорядоченный тетраэдрический набор н-граней. Рассмотрим любую точку $M$ из пересечения собственных полупространств н-граней набора $U$. Оперением $M$ в наборе $U$ назовем упорядоченную четверку $\left\langle\vec{W}_{1}, \ldots, \vec{W}_{4}\right\rangle$ векторов, выходящих из $M$ и удовлетворяющих условиям $\vec{W}_{t}=\vec{V}_{t}$, $t=1, \ldots, 4$. 
Из леммы 19 вытекает следующее свойство оперений.

Лемма 47. Пусть А и B-упорядоченные одинаково ориентированнье тетраэдрические наборы, а М и ветственно наборов А и В. Если $\left\langle\vec{V}_{1}, \ldots, \vec{V}_{4}\right\rangle$ - оперение $M$ в наборе $A$, a $\left\langle\vec{W}_{1}, \ldots, \vec{W}_{4}\right\rangle$ - оперение $H$ в наборе $B$, то $\left\langle\vec{V}_{1}, \vec{V}_{2}, \vec{V}_{3}\right\rangle u\left\langle\vec{W}_{1}, \vec{W}_{2}, \vec{W}_{3}\right\rangle$ - эквивалентные реперь.

Теперь изложим доказательство достаточности условия теоремы 3.

Лемма 48. Если $T$ и $P-$ орзвезды $и \chi(P) \leqslant \chi(T)$, то орзвезда $P$ параллельно вкладывается в орзвезду $T$.

Доказательство. Пусть $A_{T}$ и $B_{T}$ - левая и правая компоненты орзвезды $T$, а $A_{P}$ и $B_{P}$ - левая и правая компоненты орзвезды $P$. Поскольку $P-$ орзвезда, имеется точка $F$, принадлежащая пересечению собственных полупространств н-граней, входящих в компоненты $P$. Пусть $\left(v_{1}, \ldots, v_{4}\right)$ и $\left(w_{1}, \ldots, w_{4}\right)-$ следы $F$ соответственно в левой и правой компоненте звезды $P$. Заменив $k$-ю н-грань набора $A_{T}$ на ее $\left(-v_{k}\right)$-смещение, a $k$-ю н-грань набора $B_{T}$ на ее $\left(-w_{k}\right)$-смещение, $k=1, \ldots, 4$, получим упорядоченные наборы $A_{T}^{*}$ и $B_{T}^{*}$. Сопоставляя условие $\chi(P) \leqslant \chi(T)$ с леммой 46 , получаем, что $A_{T}^{*}$ и $B_{T}^{*}$ - левая и правая компоненты орзвезды $T^{*}$, параллельно вложенной в орзвезду $T$. Обозначим пересечения собственных полупространств н-граней из наборов $A_{T}$ и $B_{T}$ через $G_{A}$ и $G_{B}$, а пересечения собственных полупространств н-граней из наборов $A_{T}^{*}$ и $B_{T}^{*}-$ через $G_{A}^{*}$ и $G_{B}^{*}$. Поскольку $T^{*}-$ орзвезда, имеется точка $M$ такая, что

$$
M \in G_{A}^{*} \cap G_{B}^{*} .
$$

Так как орзвезда $T^{*}$ параллельно вложена в орзвезду $T$, справедливы включения

$$
G_{A}^{*} \subseteq G_{A}, \quad G_{B}^{*} \subseteq G_{B} .
$$

Из (32) и (33) вытекает, что точка $M$ имеет следы в каждом из наборов $A_{T}, B_{T}, A_{T}^{*}$, $B_{T}^{*}$. Пусть $\left(a_{1}, \ldots, a_{4}\right)$ и $\left(b_{1}, \ldots, b_{4}\right)$ - следы $M$ в наборах $A_{T}$ и $B_{T}$, и $\left(a_{1}^{*}, \ldots, a_{4}^{*}\right)$ и $\left(b_{1}^{*}, \ldots, b_{4}^{*}\right)$ - ее следы в наборах $A_{T}^{*}$ и $B_{T}^{*}$. Из предложения 14 и соотношений (32) и (33) получаем, что

$$
a_{k}=a_{k}^{*}+v_{k}, \quad b_{k}=b_{k}^{*}+w_{k}, \quad k=1, \ldots, 4 .
$$

Вернемся к точке $F$. Пусть оперение $F$ соответственно в левой и правой компоненте орзвезды $P$ - это упорядоченные четверки векторов $\left\langle\vec{N}_{1, P}, \ldots, \vec{N}_{4, P}\right\rangle$ и $\left\langle\vec{D}_{1, P}, \ldots, \vec{D}_{4, P}\right\rangle$. Пусть оперение точки $M$ в левой компоненте орзвезды $T$ есть упорядоченная четверка векторов $\left\langle\vec{N}_{1, T}, \ldots, \vec{N}_{4, T}\right\rangle$, а ее оперение в правой компоненте $T-$ упорядоченная четверка векторов $\left\langle\vec{D}_{1, T}, \ldots, \vec{D}_{4, T}\right\rangle$. Из определения 29 следует, что

$$
\vec{D}_{k, P}=-d_{k, P} \vec{N}_{k, P}, \quad \vec{D}_{k, T}=-d_{k, T} \vec{N}_{k, T}
$$

для некоторых $d_{k, P}>0, d_{k, T}>0, k=1, \ldots, 4$. В силу леммы 47 и предложения 16 , существует такая комбинация $\varphi$ поворота и параллельного переноса, что

$$
\begin{aligned}
\varphi(F) & =M, \\
\varphi\left(\vec{N}_{k, P}\right) & =c_{k} \vec{N}_{k, T}, \quad c_{k}>0, \quad k=1,2,3 .
\end{aligned}
$$


Из леммы 19 и предложения 11 при этом получаем, что

$$
\varphi\left(\vec{N}_{4, P}\right)=c_{4} \vec{N}_{4, T}, \quad c_{4}>0 .
$$

Преобразование $\varphi$ сохраняет линейные соотношения между векторами. Сопоставляя это с (35), (37) и (33), получаем равенства

$$
\varphi\left(\vec{D}_{k, P}\right)=u_{k} \vec{D}_{k, T}, \quad u_{k}>0, \quad k=1, \ldots, 4
$$

Преобразование $\varphi$ не меняет расстояний. Поэтому из (36) и леммы 23 получаем, что следы точки $M$ в левой и правой компонентах звезды $\varphi(P)$ совпадают со следами точки $F$ в левой и правой компоненте звезды $P$. Следовательно, следами $M$ в левой и правой компоненте звезды $\varphi(P)$ являются векторы $\left(v_{1}, \ldots, v_{4}\right)$ и $\left(w_{1}, \ldots, w_{4}\right)$. Совместно с (34) это дает следующие неравенства между следами точки $M$ в левой и правой компонентах звезды $\varphi(P)$ и ее следами $\left(a_{1}, \ldots, a_{4}\right)$ и $\left(b_{1}, \ldots, b_{4}\right)$ в левой и правой компонентах орзвезды $T$ :

$$
a_{k} \geqslant v_{k}, \quad b_{k} \geqslant w_{k}, \quad k=1, \ldots, 4 .
$$

Сопоставляя данные соотношения с (37), (38), (39) и предложением 13, получаем, что

- собственное полупространство $k$-й н-грани из упорядоченного набора $\varphi\left(A_{P}\right)$ содержится в собственном полупространстве $k$-й н-грани из упорядоченного набора $A_{T}$, $k=1, \ldots, 4$

- собственное полупространство $k$-й н-грани из упорядоченного набора $\varphi\left(B_{P}\right)$ содержится в собственном полупространстве $k$-й н-грани из упорядоченного набора $B_{T}$, $k=1, \ldots, 4$.

Но это означает, что орзвезда $\varphi(P)$ параллельно вложена в орзвезду $T$. Следовательно, орзвезда $P$ параллельно вкладывается в орзвезду $T$.

Эта лемма показывает, что условие, накладываемое в теореме 3 на характеристики, является достаточным.

\section{5. Заключение}

Естественно возникает вопрос о распространении предложенного комбинаторного подхода и результатов на класс алмазов, более широкий, чем октаэдрические. По всей видимости, это можно сделать для алмазов, близких к октаэдрическим - например, к алмазам со скругленными ребрами. Что касается возможности распространения комбинаторного подхода на еще более широкие классы алмазов, то этот вопрос нуждается в дальнейшем исследовании.

Стоит заметить, что предложенный подход к параллельному вложению с точностью до зеркального отражения октаэдрических многогранников может быть естественно распространен на пространство большего числа измерений.

Отметим также, что проблемы, связанные с алмазами, мало исследованы, хотя здесь немало трудных и нерешенных математических задач. Одна из целей этой статьи - привлечь внимание к таким вопросам. 


\section{Список литературы}

1. Фридман А. А., Бабат Л. Г., Мировой алмазобриллиантовый рынок. Математические аспекты оценки и обработки алмазов. ЦЭМИ РАН, Москва, 1995.

2. Фридман А. А., Бабат Л. Г., Мировой алмазобриллиантовый рынок. Математический метод оценки и обработки алмазов. ЦЭМИ РАН, Москва, 1995.

3. Фридман А. А., Бабат Л. Г., Мировой алмазобриллиантовый рынок. Исследование влияния формыл алмаза на его стоимость: математический подход и компьютерная реализация. ЦЭМИ РАН, Москва, 1996.

4. Фридман А. А., Бабат Л. Г., Оптимальное вложение бриллианта в алмаз и стоимостная оценка алмаза. ЦЭМИ РАН, Москва, 2005.

5. Strodiot J., Nguyen Van Hien, Nguyen Van Thoai, Application of nonsmooth optimization to a design: Centering problem. В сб.: Специальный отчет Международного института системного анализа, 1985.

Статья поступила 20.10.2006. Переработанный вариант поступил 22.03.2007. 